JOURNAL OF THE AMERICAN MATHEMATICAL SOCIETY

Volume 20, Number 4, October 2007, Pages 1053-1077

S 0894-0347(07)00564-4

Article electronically published on May 31, 2007

\title{
LOWER BOUNDS ON VOLUMES OF HYPERBOLIC HAKEN 3-MANIFOLDS
}

\author{
IAN AGOL, PETER A. STORM, AND WILLIAM P. THURSTON, \\ WITH AN APPENDIX BY NATHAN DUNFIELD
}

\section{INTRODUCTION}

In this paper we prove a volume inequality for 3-manifolds having $C^{0}$ metrics "bent" along a surface satisfying certain curvature conditions. The method is a direct generalization of work of Bray [7] and Miao [28] on the Riemannian Penrose conjecture. We also make use of Perelman's results on geometrization 34, 33. Perelman's montonicity formula for the Ricci flow with surgery (discovered by Hamilton in the case of the standard Ricci flow [18, Thm. 2.1]) implies that if $(M, g)$ is a hyperbolic 3 -manifold and $(M, h)$ is a Riemannian metric such that the scalar curvature $R(h) \geq-6=R(g)$, then $\operatorname{Vol}(M, h) \geq \operatorname{Vol}(M, g)$. More generally, if $M$ is not hyperbolic, then $\operatorname{Vol}(M, h) \geq V_{3}\|M\|$, where $V_{3}\|M\|$ denotes the simplicial volume of $M$ ( $V_{3}$ is the volume of a regular ideal tetrahedron in $\mathbb{H}^{3}$, and $\|M\|$ is the Gromov norm of the fundamental class of the 3 -manifold $M)$. This sort of curvature condition is much weaker than the conditions on Ricci curvature used in the work of Besson, Courtois, and Gallot. Their work implies a similar volume estimate if $\operatorname{Ric}(h) \geq-2 h[6$.

Theorem 7.2 is the main result of this paper, which states that if $(M, g)$ is a compact hyperbolic 3-manifold with minimal surface boundary, then $\operatorname{Vol}(M, g) \geq$ $\frac{1}{2} V_{3}\|D M\|$, where $D M$ denotes the double of $M$ along its boundary. This result has some interesting consequences. First, it implies a strong form of a conjecture of Bonahon, stating that the volume of a hyperbolic 3-manifold $M$ with convex boundary is $\geq \frac{1}{2} V_{3}\|D M\|$, with equality only in the case that $M$ has geodesic boundary. This was proven by the second author in 42, using the methods of Besson, Courtois, and Gallot [6]. Since one can find a minimal surface representing the (maximally compressed) boundary of $M$, Bonahon's conjecture follows immediately from Theorem 7.2 (see Theorem 8.2 ).

Theorem 7.2 also implies that if $M$ is a compact hyperbolic Haken 3-manifold (without boundary) and $\Sigma \subset M$ is an incompressible surface, then $\operatorname{Vol}(M) \geq$ $\frac{1}{2} V_{3}\|D(M-\mathcal{N}(\Sigma))\|$ (where $\mathcal{N}(\Sigma)$ denotes a regular neighborhood of $\Sigma$ ). This follows immediately from Theorem 7.2 by noticing that $\Sigma$ is isotopic to a minimal

Received by the editors June 30, 2005.

2000 Mathematics Subject Classification. Primary 58Jxx, 57Mxx.

The first author was partially supported by NSF grant DMS-0204142 and the Sloan Foundation.

The second author was partially supported by an NSF postdoctoral fellowship.

The third author was partially supported by the NSF grant DMS-0343694.

The last author was partially supported by the NSF grant DMS-0405491 and the Sloan foundation. 
surface in $M$. We also generalize this result to the finite volume case in Theorem 9.1. One illuminating special case is to consider a compact hyperbolic 3-manifold $M$ with geodesic boundary. If one doubles $M$, there is a natural hyperbolic structure on $D M$ induced from $M$. If one takes two copies of $M$ and glues the two copies of $\partial M$ by a diffeomorphism $\psi$ which is not isotopic to an isometry of $\partial M$, then by Thurston's hyperbolization theorem $M \cup_{\psi} M$ has a canonical hyperbolic metric. Theorem 7.2 implies that $\operatorname{Vol}\left(M \cup_{\psi} M\right)>\operatorname{Vol}(D M)$. One may compare this to gluing manifolds along tori, in which case the simplicial volume is additive [43].

Another application of our volume inequality given in section 10 is to show that the minimal volume closed orientable hyperbolic 3-manifold has volume $\geq .67$ (due to Agol and Dunfield). This improves on the previous best lower bound of .33, due to Przeworski [35. It is conjectured that the Weeks manifold, with volume $.9427 \ldots$, is the minimal volume orientable hyperbolic 3 -manifold. If $M$ is a closed hyperbolic 3-manifold with a closed embedded geodesic $\gamma$ with tube radius $R$, then we estimate the hyperbolic volume of $M-\gamma$ in terms of $\operatorname{Vol}(M)$ and $R$. This improves on the main result of 22. In the appendix, Dunfield compares these estimates with experimental data via Goodman's program tube [17.

In section 2, we state definitions and the main theorem, as well as some immediate corollaries. In section 3, we state Perelman's monotonicity results. Sections 4-7 prove the main theorem, and sections 8 and 9 extend this to the noncompact case. Section 10 proves the results on minimal volume orientable hyperbolic 3-manifolds, and section 11 discusses universal manifold pairings. Section 12 gives some open questions stemming from this work.

\section{Definitions AND STATEMENT OF THE MAIN THEOREM}

Usually, we will be assuming that manifolds are smooth, 3-dimensional, and orientable in this paper (most results for the nonorientable case follow by passing to the orientable double cover). A properly embedded incompressible surface $\Sigma^{2}$ in $M^{3}$ is a surface (with no 2-sphere or projective plane component) for which the fundamental group injects. A manifold is irreducible if every embedded 2-sphere bounds a ball. An irreducible manifold with an incompressible surface is called Haken. If a Riemannian manifold has boundary, then it is hyperbolic with totally geodesic boundary if the metric is locally modelled on a closed half-space in $\mathbb{H}^{3}$ bounded by a geodesic plane. If $\left(M^{3}, g\right)$ is a Riemannian manifold and $\Sigma^{2} \subset M$ is an embedded surface, then $M \backslash \backslash \Sigma$ denotes the Riemannian manifold with boundary obtained by taking the path metric completion of $M-\Sigma$. Here $M \backslash \backslash \Sigma$ will have a new boundary corresponding to the unit normal bundle of $\Sigma \subset M$.

The following theorem is a special case of Theorem 7.2 .

Theorem. If $(M, g)$ is a compact hyperbolic 3-manifold with minimal surface boundary, then $\operatorname{Vol}(M, g) \geq \frac{1}{2} V_{3}\|D M\|$, where $V_{3}=1.0149 \ldots$ denotes the volume of a regular ideal tetrahedron in $\mathbb{H}^{3}$.

In particular, if $M$ is acylindrical, then $M$ admits a hyperbolic metric $\nu$ with totally geodesic boundary and $\operatorname{Vol}(M) \geq \operatorname{Vol}(M, \nu)$. We conjecture that in Theorem 7.2. we do not need to assume that the boundary is compact (see Conjecture 12.3).

Let $V_{8}=3.66 \ldots$ denote the volume of a regular ideal octahedron in $\mathbb{H}^{3}$. Miyamoto showed that if $M$ is hyperbolic with totally geodesic boundary, then $\operatorname{Vol}(M) \geq$ 
$-V_{8} \chi(M)$, with equality holding only for manifolds composed of regular ideal octahedra, glued together in the pattern of an ideal triangulation [29].

Definition 2.1. If $M$ is a 3-manifold with boundary whose interior admits a hyperbolic structure, guts $(M)=N \subset M$ is a codimension-0 submanifold such that $\partial N=\partial_{0} N \cup \partial_{1} N$, where $\partial_{i} N$ is a subsurface, $\partial_{0} N=N \cap \partial M, \partial_{1} N$ consists of annuli or tori such that $\partial \partial_{1} N=\partial_{1} N \cap \partial M$. Moreover, $\left(N, \partial_{1} N\right)$ is the maximal pared acylindrical submanifold such that no components of $N$ are solid tori. One may also characterize guts $(M)=N$ by the properties that $N-\partial_{1} N$ admits a complete hyperbolic metric with totally geodesic boundary and $D(M-N)$ admits a graph manifold structure [30, 23, 24, 15].

The following result was conjectured in [1].

Theorem 9.1. Let $\bar{N}$ be a compact manifold with interior $(N, g)$, a hyperbolic 3-manifold of finite volume. Let $\bar{\Sigma}$ be an embedded incompressible ( $\pi_{1}$-injective) surface in $\bar{N}$. Then

$$
\operatorname{Vol}(N, g) \geq \frac{1}{2} V_{3}\|D(N \backslash \backslash \Sigma)\| \geq-V_{8} \chi(\operatorname{guts}(M \backslash \backslash \Sigma)) .
$$

In particular, if $(M, g)$ is a closed Haken hyperbolic 3-manifold such that $\operatorname{Vol}(M, g) \leq V_{8}$, then for any incompressible surface $\Sigma \subset M$, guts $(M \backslash \backslash \Sigma)=\emptyset$. In this case, $M \backslash \backslash \Sigma$ is a book of $I$-bundles and $\Sigma$ is called a fibroid (this improves greatly on the estimates of [11, 19]). The ramifications of this result will be pursued in subsequent papers. We record for now the following improvement on a theorem of Lackenby [26]. If $D$ is the projection of a link $L \subset S^{3}$ onto $S^{2}$, then the crossings of $D$ are divided into twist equivalence classes, where two crossings are twist equivalent if there is a loop in the projection sphere intersecting $D$ transversely precisely in the two crossings. The number of twist classes of crossings $t(D)$ is called the

twist number of $D$. One assumes that the diagram $D$ has no crossings separating D.

Theorem 2.2. Let $L \subset S^{3}$ be a hyperbolic alternating link with minimal alternating projection $D$. Then

$$
V_{8}(t(D) / 2-1) \leq \operatorname{Vol}\left(S^{3}-L\right) \leq 10 V_{3}(t(D)-1) .
$$

The lower inequality is an equality for the Borromean rings and follows directly from Theorem 5 of [26] in conjunction with Theorem 9.1. The upper inequality is given in the appendix to [26].

\section{Monotonicity formulae for the Ricci flow}

If $(M, g)$ is a closed Riemannian 3-manifold, let $\Delta_{g}=\delta d$ be the (nonpositive) Laplace operator. Let $\lambda(g)$ denote the minimal eigenvalue of the operator $-4 \Delta_{g}+$ $R(g)$, and define the scale invariant quantity

$$
V_{\lambda}(g)=\operatorname{Vol}(M, g)\left(-\frac{1}{6} \min \{\lambda(g), 0\}\right)^{3 / 2}
$$

(which is equivalent to taking the volume of the metric rescaled so that $\lambda(g)=-6$ when $\lambda(g)<0)$. Recall that $V_{3}\|M\|$ is the sum of the volumes of the hyperbolic 
pieces of the geometric decomposition of $M$ 43. If $R_{\min }(g) \geq 0$ (where $R_{\min }(g)$ is the minimum of $R(g))$, then $\|M\|=0$ [33, Thm. 8.2]. Also, define

$$
V_{R}(g)=\operatorname{Vol}(M, g)\left(-\frac{1}{6} \min \left\{R_{\min }(g), 0\right\}\right)^{3 / 2} .
$$

We have the inequality $\lambda(g) \geq R_{\min }(g)$, since $-4 \Delta_{g}+R \geq-4 \Delta_{g}+R_{\min } \geq R_{\min }$, and thus $V_{R}(g) \geq V_{\lambda}(g)$. Perelman showed that $V_{R}(g)$ and $V_{\lambda}(g)$ are monotonic decreasing for metrics evolving by the Ricci flow with surgery (monotonicity of $V_{R}(g)$ for the Ricci flow was shown by Hamilton [18, Thm. 2.1]). Then Perelman's analysis of the geometric decomposition forming at infinite time under Ricci flow with surgery implies the following theorem (a restatement of Theorem 8.2 [33]).

Theorem 3.1. Let $(M, g)$ be a closed 3-manifold. Then

$$
V_{R}(g) \geq V_{\lambda}(g) \geq V_{3}\|M\| \text {. }
$$

It is much simpler to prove that $V_{R}(g)$ is monotonic decreasing with respect to Ricci flow with surgery than to prove the monotonicity of $V_{\lambda}(g)$. The monotonicity formula for $V_{\lambda}(g)$ through surgeries is given in [25, Proposition 92.4]. The monotonicity of $V_{R}(g)$ through surgery is obvious from Perelman's formulation of surgery, since the surgery decreases volume and does not change $\min \left\{R_{\min }(g), 0\right\}$, since the surgeries occur at a part of the manifold where $R(g) \gg 0$. Thus, in the proof of Theorem 7.2. we give two different arguments, one estimating $\lambda(g)$ and the other performing a conformal change to estimate $R_{\min }(g)$. Although the authors of this paper have not checked the validity of Perelman's papers, there are references available which work out many of the details of Perelman's arguments. Also, a key aspect of Perelman's geometrization proof is a collapsing theorem which he has not made available. Since this is a key to the above Theorem 3.1, we note that Shioya and Yamaguchi have a paper 39] which claims to give an alternative proof of the collapsing results needed by Perelman. See also [10, 25, 31].

\section{Smoothing With a LOWER SCALAR CURVATURe Bound}

This section produces smooth Riemannian approximations to a particular kind of singular Riemannian metric while maintaining a lower bound on the scalar curvature. The main result of this section is Proposition 4.2 which will be used as an input to Theorem 7.1. In a different setting, this smoothing procedure was developed as a tool to solve the Riemannian Penrose conjecture in general relativity (see Bray [7] and Miao [28]). The techniques of this section are adaptations of their methods to the present situation.

Let $(M, G)$ be a compact 3-manifold with boundary and smooth Riemannian metric $G$, such that $\partial M=\Sigma_{-} \cup \Sigma_{+}$. Assume that there is an isometry $\Phi: \Sigma_{+} \rightarrow$ $\Sigma_{-}$, satisfying the following curvature condition for all $x \in \Sigma_{+}$:

$$
H\left(\Sigma_{+}, G\right)(x)+H\left(\Sigma_{-}, G\right)(\Phi(x)) \geq 0,
$$

where $H$ is the mean curvature with respect to the outward pointing normal. Let

$$
(X, g)=(M /\{x \sim \Phi(x)\}, G)
$$

be the closed smooth manifold obtained by gluing $\Sigma_{+}$to $\Sigma_{-}$by the isometry $\Phi$. The metric tensor $g$ is a piecewise smooth Riemannian metric on $X$. In particular, $g$ is a Lipschitz Riemannian metric (this means that in the $C^{\infty}$ structure on $X$, in any chart the metric $g$ will be a Lipschitz function on $\mathbb{R}^{3}$ ). We will use $\Sigma \subset X$ to 
denote the surface formed by identifying the boundary components $\Sigma_{ \pm}$of $M$. Fix a collared tubular neighborhood $\Sigma \times\left(-\delta_{0}, \delta_{0}\right) \hookrightarrow X$ taking $\Sigma \times\{0\}$ to $\Sigma \subset X$, so that the second coordinate is the distance function to $\Sigma$ (Fermi coordinates).

Two relevant examples are: $M$ consists of two copies of a hyperbolic 3-manifold with minimal surface boundary or a finite volume hyperbolic 3-manifold truncated along a tube about a geodesic together with a horospherical cusp of appropriate shape. It might be possible to interpret the inequality in equation (1) in terms of a distributional positive scalar curvature concentrated along the surface $\Sigma$, but we have not yet been able to achieve this.

Under the above hypotheses, we paraphrase the following result of Miao:

Proposition 4.1 ([28, Proposition 3.1]). Suppose that $(X, g)$ is defined by equation (22), satisfying the conditions of equation (11). There exists a family of smooth Riemannian metrics $\left\{g_{\delta}\right\}_{0<\delta<\delta_{0}}$ on $X$ so that $g_{\delta}$ converges uniformly to $g$ as $\delta \rightarrow 0$, the metrics $g_{\delta}$ and $g$ agree outside $\Sigma \times(-\delta / 2, \delta / 2)$, and the scalar curvature $R\left(g_{\delta}\right)$ is bounded below by a constant $\mathcal{S}$ independent of $\delta$.

Remark. Miao performs the smoothing via a mollification method. In the main case of interest in this paper, which has an extra symmetry, one may use the more elementary method of Bray [7, Thm. 9].

Stated more geometrically, the fact that the metrics $g_{\delta}$ converge uniformly to $g$ implies that the Lipschitz constant of the identity map $\left(X, g_{\delta}\right) \rightarrow(X, g)$ converges to one. In particular, the volume of $\left(X, g_{\delta}\right)$ converges to the volume of $(X, g)$.

The goal of this section is to prove

Proposition 4.2. Assume that $R(G) \geq-6$. Then there exists a family of smooth Riemannian metrics $G_{\delta}$ on $X$ such that the metrics $G_{\delta}$ converge uniformly to $g$ and have scalar curvature $R\left(G_{\delta}\right)$ bounded below by -6 . In particular, $\operatorname{Vol}\left(X, G_{\delta}\right)$ converges to $\operatorname{Vol}(X, g)$.

The metrics $G_{\delta}$ will be obtained by conformally altering the smooth metrics $g_{\delta}$ produced in Proposition 4.1. The method is an adaptation of techniques of Bray [7] and Miao 28] dealing with the case of open asymptotically flat manifolds of nonnegative scalar curvature. We will choose functions $u_{\delta}$, such that $G_{\delta}=u_{\delta}^{4} g_{\delta}$. It is known that under such a conformal change, the scalar curvature of the new metric is given by the formula

$$
R\left(G_{\delta}\right)=u_{\delta}^{-5}\left(-8 \Delta_{\delta}+R\left(g_{\delta}\right)\right) u_{\delta}
$$

38. To control $R\left(G_{\delta}\right)$, we will choose $u_{\delta}$ to be an eigenfunction of a carefully chosen elliptic operator. In some sense, this method diffuses the scalar curvature $<-6$ near $\Sigma$ across the manifold $X$.

Before beginning the proof, we note that if for all sufficiently small $\delta, R\left(g_{\delta}\right) \geq-6$, then we are done. So we can assume without loss of generality that $R\left(g_{\delta}\right) \geq \mathcal{S}$ and $\mathcal{S}<-6$, where $\mathcal{S}$ is independent of $\delta$ by Proposition 4.1.

Define a smooth function $\mathcal{R}_{\delta}: X \longrightarrow[\mathcal{S}+6,0]$ such that $\mathcal{R}_{\delta}$ restricted to $\Sigma \times(-\delta / 2, \delta / 2)$ is exactly $\mathcal{S}+6$ and $\mathcal{R}_{\delta}$ vanishes outside $\Sigma \times(-\delta, \delta)$. Note that the $g_{\delta}$-volume of the support of $\mathcal{R}_{\delta}$ converges to zero as $\delta \rightarrow 0$.

Define the operator $-8 \Delta_{\delta}+\mathcal{R}_{\delta}$, where $\Delta_{\delta}$ is the Laplacian for the metric $g_{\delta}$. This Schrödinger operator has a minimal eigenvalue $\lambda_{\delta}$ with a strictly positive smooth 
eigenfunction $u_{\delta}$ [36, XIII.12]. Let us normalize the eigenfunction so that

$$
\int_{X} u_{\delta}^{2} d g_{\delta}=\operatorname{Vol}\left(X, g_{\delta}\right)
$$

If $\lambda_{\delta}$ were nonnegative, then the eigenfunction would satisfy

$$
-8 \Delta u_{\delta}=\lambda_{\delta} u_{\delta}-\mathcal{R}_{\delta} u_{\delta} \geq 0,
$$

making $u_{\delta}$ a superharmonic function. Since $X$ is compact and $\mathcal{R}_{\delta}$ is not a constant function, this is impossible. Therefore $\lambda_{\delta}<0$. Take the equation

$$
-8 \Delta u_{\delta}+\mathcal{R}_{\delta} u_{\delta}=\lambda_{\delta} u_{\delta}
$$

multiply it by $u_{\delta}$, integrate over $X$, and perform integration by parts to obtain

$$
\int_{X}\left(8\left|\nabla u_{\delta}\right|^{2}+\mathcal{R}_{\delta} u_{\delta}^{2}\right) d g_{\delta}=\lambda_{\delta} \operatorname{Vol}\left(X, g_{\delta}\right)<0
$$

This yields the estimate

$$
\int_{X}\left|\nabla u_{\delta}\right|^{2} d g_{\delta}<\frac{1}{8} \int_{X}\left|\mathcal{R}_{\delta}\right| u_{\delta}^{2} d g_{\delta} \leq \frac{|\mathcal{S}+6|}{8} \operatorname{Vol}\left(X, g_{\delta}\right) .
$$

The following lemma is based on the method of Proposition 4.1 [28].

Lemma 4.3. $\int_{X} \mathcal{R}_{\delta} u_{\delta}^{2} d g_{\delta} \longrightarrow 0$ as $\delta \rightarrow 0$.

Proof. Note that $\left|\mathcal{R}_{\delta}\right|$ is uniformly bounded by $|\mathcal{S}+6|$. Apply Hölder's inequality to obtain

$$
\begin{aligned}
\int_{X}\left|\mathcal{R}_{\delta}\right| u_{\delta}^{2} d g_{\delta} & \leq\left(\int_{X}\left|\mathcal{R}_{\delta}\right|^{3 / 2} d g_{\delta}\right)^{2 / 3} \cdot\left(\int_{X} u_{\delta}^{6} d g_{\delta}\right)^{1 / 3} \\
& \leq|\mathcal{S}+6|\left(\operatorname{Vol}\left(\operatorname{supp}\left(\mathcal{R}_{\delta}\right), g_{\delta}\right)\right)^{2 / 3}\left\|u_{\delta}\right\|_{6}^{2}
\end{aligned}
$$

If we can show that $\left\|u_{\delta}\right\|_{6}$ is uniformly bounded, then the term to the right goes to zero, since the volume of the support of $\mathcal{R}_{\delta}$ goes to zero. We now apply the Sobolev inequality (see e.g. [4, Thm. 2.21]) to obtain

$$
\left\|u_{\delta}\right\|_{6} \leq C\left(\left\|\nabla u_{\delta}\right\|_{2}+\left\|u_{\delta}\right\|_{2}\right)<C \operatorname{Vol}\left(X, g_{\delta}\right)^{\frac{1}{2}}\left((|\mathcal{S}+6| / 8)^{\frac{1}{2}}+1\right) .
$$

Note that the uniform convergence of the metrics $g_{\delta}$ to $g$ implies we can use a single Sobolev constant $C$ for all the metrics $g_{\delta}$. The parts of the Sobolev inequality which depend on the metric are the volume form which is used to compute the various norms and $\nabla u_{\delta}$, which is the dual vector field to the 1 -form $d u_{\delta}$, under the identification $g_{\delta}: T^{*} X \rightarrow T X$. Both of these terms depend continously on the metric $g_{\delta}$, which implies that we may choose a uniform Sobolev constant $C$ for the metrics $g_{\delta}$. Therefore the number on the right hand side of the Sobolev inequality is uniformly bounded for all $\delta$, which completes the proof that $\left\|u_{\delta}\right\|_{6}$ is bounded.

This lemma has two corollaries.

\section{Corollary 4.4.}

$$
\int_{X}\left|\nabla u_{\delta}\right|^{2} d g_{\delta} \longrightarrow 0 \quad \text { and } \quad \lambda_{\delta} \rightarrow 0
$$

as $\delta \rightarrow 0$.

Proof. Since $\left|\nabla u_{\delta}\right|^{2} \geq 0$, the first part of the corollary follows from the lemma and equation (4), and the second part follows from the first part, the lemma, and equation (3). 
Define the average

$$
\bar{u}_{\delta}:=\frac{1}{\operatorname{Vol}\left(X, g_{\delta}\right)} \int_{X} u_{\delta} d g_{\delta}
$$

\section{Corollary 4.5.}

$$
\int\left(u_{\delta}-\bar{u}_{\delta}\right)^{2} d g_{\delta} \rightarrow 0, \quad \bar{u}_{\delta} \rightarrow 1
$$

as $\delta \rightarrow 0$.

Proof. The Poincaré inequality for $\left(X, g_{\delta}\right)$ yields

$$
\int_{X}\left(u_{\delta}-\bar{u}_{\delta}\right)^{2} d g_{\delta} \leq C \int_{X}\left|\nabla u_{\delta}\right|^{2} d g_{\delta}
$$

In fact, one may take $C=1 / \lambda_{1}(X)$, where $\lambda_{1}(X)$ is the minimal nonzero eigenvalue of $-\Delta$ on $X$, by the Rayleigh characterization of $\lambda_{1}(X)$. The metrics $g_{\delta}$ converge uniformly to $g$, implying we may use a uniform constant $C$ in the Poincaré inequality for all $\delta$ (see the justification given in Lemma 4.3 for a uniform Sobolev constant). From this the first limit of the corollary follows.

A short computation yields

$$
\int_{X}\left(u_{\delta}-\bar{u}_{\delta}\right)^{2} d g_{\delta}=\operatorname{Vol}\left(X, g_{\delta}\right)-\operatorname{Vol}\left(X, g_{\delta}\right) \cdot \bar{u}_{\delta}^{2}
$$

The second limit of the corollary follows from this and the previous paragraph.

Define

$$
w_{\delta}:=u_{\delta}-\bar{u}_{\delta} .
$$

From the fact that $u_{\delta}$ is an eigenfunction we obtain the following equation for $w_{\delta}$ :

$$
-8 \Delta_{\delta} w_{\delta}+\left(\mathcal{R}_{\delta}-\lambda_{\delta}\right) w_{\delta}=\left(\lambda_{\delta}-\mathcal{R}_{\delta}\right) \bar{u}_{\delta} .
$$

As above, the Sobolev inequality

$$
\left\|w_{\delta}\right\|_{6} \leq C\left(\left\|\nabla u_{\delta}\right\|_{2}+\left\|w_{\delta}\right\|_{2}\right)
$$

can be applied with a uniform constant $C$ for all the metrics $g_{\delta}$. By the above corollaries, the right hand side goes to 0 , so

$$
\left\|w_{\delta}\right\|_{6} \longrightarrow 0
$$

Using equation (5), it follows from the theory of elliptic partial differential equations [4. Thm. 4.42] that

$$
\sup _{X}\left|w_{\delta}\right| \leq C_{2}\left\{\left(\int_{X} w_{\delta}^{6} d g_{\delta}\right)^{1 / 6}+\left(\int_{X} \bar{u}_{\delta}^{3}\left|\lambda_{\delta}-\mathcal{R}_{\delta}\right|^{3} d g_{\delta}\right)^{1 / 3}\right\} \longrightarrow 0
$$

as $\delta \rightarrow 0$. The theorem cited [4, Thm. 4.42] is stated locally, but it gives a global estimate by taking an open cover by charts for $X$. It is again worth noting that a single constant $C_{2}$ is valid for all the metrics $g_{\delta}$ because they converge uniformly to $g$. (Written in divergence form, the derivatives of the metric tensor do not appear in the coefficients of the local expression of the second order elliptic PDE satisfied by $w_{\delta}$. So nothing is blowing up as $\delta \rightarrow 0$.) We have shown that $u_{\delta} \rightarrow \bar{u}_{\delta}$ and $\bar{u}_{\delta} \rightarrow 1$ uniformly as $\delta \rightarrow 0$.

We may now define a new family of metrics $\widetilde{g}_{\delta}$ on $X$ by the conformal change

$$
\widetilde{g}_{\delta}:=u_{\delta}^{4} \cdot g_{\delta} .
$$


It is known that under such a conformal change, the scalar curvature of the new metric is given by the formula

$$
R\left(\widetilde{g}_{\delta}\right)=u_{\delta}^{-5}\left(-8 \Delta_{\delta}+R\left(g_{\delta}\right)\right) u_{\delta}
$$

38. Since $u_{\delta}$ is an eigenfunction, we have the equation

$$
\begin{aligned}
\left(-8 \Delta_{\delta}+R\left(g_{\delta}\right)\right) u_{\delta} & =\left(\lambda_{\delta}-\mathcal{R}_{\delta}\right) u_{\delta}+R\left(g_{\delta}\right) u_{\delta} \\
& =\left(R\left(g_{\delta}\right)-\mathcal{R}_{\delta}\right) u_{\delta}+\lambda_{\delta} u_{\delta} .
\end{aligned}
$$

By the construction of the function $\mathcal{R}_{\delta} \geq \mathcal{S}+6$, it follows that $R\left(g_{\delta}\right)-\mathcal{R}_{\delta}$ is everywhere bounded below by -6 . Since $\lambda_{\delta} \rightarrow 0$ and $u_{\delta} \rightarrow 1$ uniformly as $\delta \rightarrow 0$, we may conclude that the scalar curvature of the metric $\widetilde{g}_{\delta}$ is bounded below by a constant converging to -6 as $\delta \rightarrow 0$.

Finally let us homothetically expand each metric $\widetilde{g}_{\delta}$ by a factor $\eta_{\delta}$, for $\eta_{\delta} \rightarrow 1$ as $\delta \rightarrow 0$, so that the resulting homothetically expanded Riemannian metrics have scalar curvature bounded below by -6 . Let us call this final family of metrics $G_{\delta}=\eta_{\delta} \cdot \widetilde{g}_{\delta}$. Notice that the metrics $G_{\delta}$ converge uniformly to $g$ as $\delta \rightarrow 0$. In particular, the volumes $\operatorname{Vol}\left(X, G_{\delta}\right)$ converge to the original $\operatorname{Vol}(X, g)=\operatorname{Vol}(M, G)$ as $\delta \rightarrow 0$. This finishes the proof of Proposition 4.2 .

\section{BOUNDING $\lambda\left(g_{\delta}\right)$}

This section gives an alternative to the argument in section 4 by showing that we may approximate $(X, g)$ (constructed at the beginning of section 4) by metrics with $\lambda\left(g_{\delta}\right) \geq-6$ rather than $R\left(G_{\delta}\right) \geq-6$. As mentioned in section 3, we are including these two alternatives since the monotonicity of $V_{R}(g)$ under Ricci flow with surgery is much simpler to prove than the monotonicity of $V_{\lambda}(g)$.

Let $(X, g)$ be the metric constructed from equation (2) and satisfying equation (11) from section 4 . Let $g_{\delta}$ be the smooth metrics approximating the metric $(X, g)$ from Proposition 4.1, such that in $\Sigma \times[-\delta / 2, \delta / 2], R\left(g_{\delta}\right)=R_{\delta} \geq \mathcal{S}$, and in $X-$ $(\Sigma \times[-\delta / 2, \delta / 2]), R_{\delta} \geq-6$, where $\mathcal{S}$ comes from Proposition 4.1. Let $\lambda(g)$ denote the minimal eigenvalue of the operator $-4 \Delta_{g}+R(g)$.

Theorem 5.1. $\liminf _{\delta \rightarrow 0} \lambda\left(g_{\delta}\right) \geq-6$.

Proof. If $\mathcal{S} \geq-6$, then the result follows directly from the fact that $\lambda\left(g_{\delta}\right) \geq \inf R_{\delta}$, so we may assume that $\mathcal{S}<-6$. Let $u_{\delta}$ be the eigenfunction of $-4 \Delta_{g_{\delta}}+R_{\delta}$ with minimal eigenvalue $\lambda\left(g_{\delta}\right)<0$, such that $u_{\delta}$ is strictly positive and $\left\|u_{\delta}\right\|_{2}=1$. We can use the Rayleigh quotient method to estimate $\lambda\left(g_{\delta}\right)$ from below. We may estimate

$$
\begin{aligned}
& \lambda\left(g_{\delta}\right)=\int_{X}\left(\left|\nabla_{\delta} u_{\delta}\right|^{2}+R_{\delta} u_{\delta}^{2}\right) d g_{\delta} \geq \int_{X-(\Sigma \times[-\delta, \delta])} R_{\delta} u_{\delta}^{2} d g_{\delta}+\int_{\Sigma \times[-\delta, \delta]} R_{\delta} u_{\delta}^{2} d g_{\delta} \\
& \geq-6 \int_{X-(\Sigma \times[-\delta, \delta])} u_{\delta}^{2} d g_{\delta}+\mathcal{S} \int_{\Sigma \times[-\delta, \delta]} u_{\delta}^{2} d g_{\delta}=-6+(\mathcal{S}+6) \int_{\Sigma \times[-\delta, \delta]} u_{\delta}^{2} d g_{\delta} .
\end{aligned}
$$

Thus, we need only show that $\int_{\Sigma \times[-\delta, \delta]} u_{\delta}^{2} d g_{\delta} \rightarrow 0$ as $\delta \rightarrow 0$. Applying Holder's inequality, we see that

$$
\begin{aligned}
\int_{\Sigma \times[-\delta, \delta]} u_{\delta}^{2} d g_{\delta} & \leq\left(\int_{\Sigma \times[-\delta, \delta]} d g_{\delta}\right)^{\frac{2}{3}}\left(\int_{\Sigma \times[-\delta, \delta]} u_{\delta}^{6} d g_{\delta}\right)^{\frac{1}{3}} \\
& =\operatorname{Vol}\left(\Sigma \times[-\delta, \delta], g_{\delta}\right)^{\frac{2}{3}}\left\|u_{\delta}\right\|_{6}^{2}
\end{aligned}
$$


The right hand term converges to 0 , since $\operatorname{Vol}\left(\Sigma \times[-\delta, \delta], g_{\delta}\right) \rightarrow 0$ as $\delta \rightarrow 0$ and $\left\|u_{\delta}\right\|_{6}$ is bounded by the following. Since the metrics $g_{\delta}$ approximate $g$ in $C^{0}$ norm, there is a uniform Sobolev constant $C$ (see the justification in Lemma 4.3) such that

$$
\left\|u_{\delta}\right\|_{6} \leq C\left(\left\|\nabla_{\delta} u_{\delta}\right\|_{2}+\left\|u_{\delta}\right\|_{2}\right) \leq C\left(\left(\lambda\left(g_{\delta}\right)-\mathcal{S}\right)^{\frac{1}{2}}+1\right) \leq C\left((-\mathcal{S})^{\frac{1}{2}}+1\right) .
$$

Rescaling the metrics $g_{\delta}$ by factors converging to 1 , we may approximate $(X, g)$ by metrics $g_{\delta}^{\prime}$ such that $\lambda\left(g_{\delta}^{\prime}\right) \geq-6$.

\section{RiCCI FLOW WITH $C^{0}$ INPUT}

Let $(X, g)$ be the smooth closed 3-manifold equipped with the Lipschitz Riemannian metric $g$ from the beginning of section 4, defined by equation (2) and satisfying the curvature conditions (11). Let $\left\{g_{\delta}\right\}_{\delta>0}$ be a family of smooth Riemannian metrics on $X$ converging uniformly to $g$. We do not yet place any other geometric assumptions on the approximates $g_{\delta}$. The goal of this section is to establish facts about flowing the singular metric $g$ in the direction of its Ricci curvature.

The analytic tools of this section were proven by M. Simon [40, where the dual Ricci harmonic map heat flow is studied with nonsmooth initial data. To apply Simon's machinery, it is necessary to fix a "background metric". Concretely this means fixing a smooth Riemannian metric $h$ on $X$ so that the identity map $(X, h) \longrightarrow(X, g)$ is $K$-bilipschitz, where $K>1$ is a fixed constant depending only on the dimension. For our purposes, it would suffice to pick a metric $g_{\delta}$ for sufficiently small $\delta$. Let us choose such a metric and call it $h$. The following theorem follows from statements in section 5 of [40].

Theorem 6.1. There exists a constant $T>0$ depending only on the background metric $h$, a sequence $\delta_{j} \rightarrow 0$, a sequence of families of smooth metrics $\left\{g\left(\delta_{j}, t\right)\right\}, 0 \leq$ $t \leq T, j \in \mathbb{N}$, and a family of metrics $g(0, t), 0 \leq t \leq T$, such that the following hold.

(1) The metric $g(0,0)=g$ and $g\left(\delta_{j}, 0\right)=g_{\delta_{j}}$.

(2) The family $\left\{g\left(\delta_{j}, t\right)\right\}_{0 \leq t \leq T}$ is conjugate via diffeomorphisms of $X$ to a solution to the Ricci flow equation with initial metric $g\left(\delta_{j}, 0\right)$. Similarly, for any $t_{0}>0$ the family $\{g(0, t)\}_{t_{0} \leq t \leq T}$ is conjugate via diffeomorphisms of $X$ to a solution to the Ricci flow equation with initial metric $g\left(0, t_{0}\right)$.

(3) $g(0, t)$ converges uniformly to $g(0,0)=g$ as $t \rightarrow 0$.

(4) $g\left(\delta_{j}, t\right)$ converges in the $\mathcal{C}^{\infty}$ sense to $g\left(\delta_{j}, 0\right)$ as $t \rightarrow 0$.

(5) For $t>0, g\left(\delta_{j}, t\right)$ converges in the $\mathcal{C}^{\infty}$ sense to $g(0, t)$ as $j \rightarrow \infty$.

Proof. Properties (1), (2), and (3) above are stated in the proof of Theorem 5.2 of 40. Property (4) is a restatement of Lemma 5.1 of [40. Also in Theorem 5.2 of [40], it is proven that there exist constants $\left\{c_{i}\right\}$ depending on $h$ such that

$$
\sup _{X}\left|\nabla^{i} g\left(\delta_{j}, t\right)\right|^{2} \leq c_{i} / t^{i},
$$

where $\nabla^{i}$ denotes the $i^{\text {th }}$ covariant derivative with respect to the background metric $h$. (By fixing a finite set of coordinate charts covering $X$ and choosing a precompact domain in each chart whose union still covers $X$, we can replace $\nabla^{i}$ with honest spatial derivatives in each coordinate chart. This will change the constants $c_{i}$ depending on how we choose our cover. This is possible because on each precompact 
domain the identity map from the metric $h$ to the Euclidean metric will be bilipschitz.) Notice that the constants of this inequality do not depend on $\delta_{j}$. For any $t_{0}>0$ this independence yields uniform control on the spatial derivatives of the metrics $g\left(\delta_{j}, t\right)$ for all $j$ and $t \geq t_{0}$. We may therefore apply the Arzela-Ascoli theorem repeatedly to deduce property (5) above. (This is in fact how Simon produces the family $g(0, t)$. See the first paragraph of the proof of Theorem 5.2 of [40].)

Recall that for a smooth Riemannian metric $h, \lambda(h)$ denotes the minimal eigenvalue of the operator $-4 \Delta_{h}+R(h)$.

Corollary 6.2. If for some $\mathcal{S} \in \mathbb{R}$ and all $j \in \mathbb{N}$ the metric $g_{\delta_{j}}$ has $\lambda\left(g_{\delta_{j}}\right)$ bounded below by $\mathcal{S}$, then for all $t>0$ the Riemannian metric $g(0, t)$ has eigenvalue $\lambda(g(0, t)) \geq \mathcal{S}$.

Proof. Perelman has shown that the eigenvalue $\lambda$ is nondecreasing along the Ricci flow (see Corollary 1.2 and Section 2.2 of [34). Therefore by properties (2) and (5) of Theorem 6.1, together with continuity of $\lambda$ in the $\mathcal{C}^{\infty}$ topology on metrics, we can deduce the statement of the corollary.

Corollary 6.3. If for all $j \in \mathbb{N}$ the metrics $g_{\delta_{j}}$ have scalar curvature bounded below by -6 , then for all $t>0$ the Riemannian metric $g(0, t)$ has scalar curvature bounded below by -6 .

Proof. The minimum scalar curvature is nondecreasing along the Ricci flow. Therefore the corollary follows from Theorem 6.1.

Remark. Theorem 6.6 of [40] shows that a manifold with a Lipschitz metric and Alexandrov positive curvature which is bounded above and below may be approximated by smooth manifolds with positive curvature. Simon mentions at the end of Theorem 6.6 that one may prove a similar statement for positive scalar curvature. But his theorem is weaker than Corollary 6.3, since we do not assume that the metric may be approximated by smooth metrics with uniformly bounded curvature. The advantage of dealing with scalar curvature (or $\lambda$ ) is that it is monotonic under the Ricci flow, whereas almost positive curvature is not, which accounts for our weaker hypotheses.

\section{MAin IneQUality}

Before we state the next theorem, recall that -6 is the scalar curvature of a 3 -manifold with constant sectional curvature -1 .

Theorem 7.1. Let $(M, g)$ be a compact 3-manifold with boundary and smooth Riemannian metric $g$, such that $R(g) \geq-6$ and $\partial M=\Sigma_{-} \cup \Sigma_{+}$. Assume that there is an isometry $\Phi: \Sigma_{+} \rightarrow \Sigma_{-}$, satisfying the following curvature condition for all $x \in \Sigma_{+}$:

$$
H\left(\Sigma_{+}, g_{+}\right)(x)+H\left(\Sigma_{-}, g_{-}\right)(\Phi(x)) \geq 0
$$

where $H$ is the mean curvature with respect to the outward pointing normal. Let $(X, g)=(M /\{x \sim \Phi(x)\}, g)$ be the closed manifold obtained by gluing $\Sigma_{+}$to $\Sigma_{-}$by the isometry $\Phi$. Then

$$
\operatorname{Vol}(X, g) \geq V_{3}\|X\|
$$

Equality holds if and only if $X$ has constant sectional curvature -1 . 
Proof. We present two slightly different proofs, one using scalar curvature and the other using the eigenvalue $\lambda(h)$ of the operator $-4 \Delta_{h}+R(h)$. Let us discuss the scalar curvature proof first.

By Proposition 4.2, there is a family of smooth metrics $\left\{g_{\delta}\right\}$ on $X$ converging uniformly to $g$ with scalar curvature bounded below by -6 . Now let $g(t)$ be the family of metrics produced by Theorem 6.1 satisfying the properties: as $t \rightarrow 0$ the metrics $g(t)$ converge uniformly to $g$, and for any $t_{0}>0$ the family $\left\{g_{t}\right\}_{t_{0} \leq t \leq T}$ is diffeomorphism-conjugate to a solution to the Ricci flow with initial metric $g_{t_{0}}$. By Corollary 6.3. for any $t>0, R_{\min }\left(g_{t}\right) \geq-6$. We may assume $R_{\min }\left(g_{t}\right)<0$ for all t. Otherwise we are done since if $R\left(g_{t}\right) \geq 0$, then $\|X\|=0$ [33, Thm. 8.2]. By Theorem 3.1, $V_{R}\left(g_{t}\right) \geq V_{3}\|X\|$. Taking the initial time $t_{0}$ down to 0 and using the fact that $\liminf _{t \rightarrow 0} V_{R}\left(g_{t}\right) \leq \operatorname{Vol}(X, g)$ by Corollary 6.3 yields the desired inequality

$$
\operatorname{Vol}(X, g) \geq V_{3}\|X\|
$$

In this argument, we may replace $V_{R}\left(g_{t}\right)$ with $V_{\lambda}\left(g_{t}\right)$, and use Corollary 6.2 instead of Corollary 6.3.

Let us now assume that $\operatorname{Vol}(X, g)=V_{3}\|X\|$. Then it follows that the quantity $V_{R}\left(g_{t}\right)$ is constant for all time. This can only occur if all the metrics $\left\{\left(X, g_{t}\right)\right\}_{t>0}$ have constant Ricci curvature [18, Sec.2] (one may also use that $V_{\lambda}\left(g_{t}\right)$ is constant, and one may use [34, Sec.2.3] and [33, Sec.8]). In three dimensions, constant Ricci curvature implies constant sectional curvature. By the uniform continuity of the metrics $g_{t}$ back to time zero, this implies that $(X, g)$ is isometric to a smooth Riemannian manifold of constant sectional curvature -1 .

Theorem 7.2. Let $(M, g)$ be a compact 3-manifold with a smooth Riemannian metric $g$ such that the scalar curvature of $g$ is at least -6 at every point and the boundary of $M$ is a minimal surface. Then

$$
\operatorname{Vol}(M, g) \geq \frac{1}{2} V_{3}\|D M\| .
$$

Equality holds if and only if $M$ has constant sectional curvature -1 and the boundary of $M$ is totally geodesic.

Proof. Let $(M \times\{+,-\}, g)$ be two identical copies of $(M, g)$, with boundary components $\Sigma_{ \pm}=\partial M \times \pm$ and isometry $\Phi: \Sigma_{+} \rightarrow \Sigma_{-}$defined by $\Phi(x,+)=(x,-)$ and $D M=(M \times\{+,-\}) / \Phi$. Since $H\left(\Sigma_{ \pm}, g_{ \pm}\right)(x)=0$, the curvature inequality hypothesis of Theorem 7.1 is satisfied. Thus, $\operatorname{Vol}(M)=\frac{1}{2} V_{3}\|D M\|$. If we have equality, then the metric on $D M$ is hyperbolic. This implies that $\partial M$ is totally geodesic, since the metric on $D M$ will be singular if the second fundamental form of $\partial M$ is nonzero anywhere.

\section{Hyperbolic CONVEX CORES}

In this section we will use Theorem 7.2 to study convex cores of hyperbolic 3manifolds. More specifically we can quickly prove a conjecture of Bonahon stating that the volume of a compact hyperbolic 3-manifold $M$ with nonempty convex boundary is at least half the simplicial volume of the doubled manifold $D M$. In the incompressible boundary setting this was proved in [42]. We will also prove the relative version of the inequality for cusped hyperbolic 3-manifolds. 
If $N$ is a complete (infinite volume) hyperbolic 3-manifold without boundary, then the convex core $C_{N} \subseteq N$ is defined to be the minimal closed convex submanifold of $N$ for which inclusion is a homotopy equivalence. The volume of the convex core $C_{N}$ is a measure of the geometric complexity of $N$. When it has infinite volume, then $N$ is said to be geometrically infinite. Otherwise $N$ is geometrically finite. This is the case of interest here.

We need some standard geometric properties of the convex core which can be found in [12.

The map $\pi: N-C_{N} \rightarrow \partial C_{N}$ given by projecting to the nearest point of the convex core is distance decreasing. The boundary of the convex core is not in general smooth, but it is always totally geodesic outside some compact set. The relationship between the topology of $N$ and $C_{N}$ is straightforward: if $N$ is geometrically finite, then it is homeomorphic to the interior of $C_{N}$.

To begin, we will prove the desired volume inequality in the compact setting.

Theorem 8.1. Let $N$ be a complete hyperbolic 3-manifold without boundary. Assume the convex core $C_{N}$ of $N$ is compact. It then satisfies the volume inequality

$$
\operatorname{Vol}\left(C_{N}\right) \geq \frac{1}{2} V_{3}\left\|D C_{N}\right\|
$$

Moreover, if $2 \operatorname{Vol}\left(C_{N}\right)=V_{3}\left\|D C_{N}\right\|$, then $C_{N}$ is a compact manifold with totally geodesic boundary.

Proof. Consider the (possibly disconnected) compact embedded surface given by the boundary of the convex core. By Dehn's lemma we can compress a finite number of homotopically trivial curves in $\partial C_{N}$ to obtain a $\pi_{1}$-injective embedded surface $S^{\prime}$ bounding a submanifold $M^{\prime}$. Throw away components $C$ of $M^{\prime}$ such that $\|D C\|=0$ (i.e. $D C$ is a graph manifold) to get a submanifold $M^{\prime \prime}$ with boundary $S^{\prime \prime}$. It follows that $\left\|D M^{\prime \prime}\right\|=\left\|D C_{N}\right\|$, by [43, Ch. 6], 41]. Using the distance decreasing map $\pi$ to push wayward surfaces back into the convex core, one can produce an embedded minimal surface $S \subset C_{N}$ isotopic to $S^{\prime \prime}$ [13, 21]. Here $S$ bounds a (possibly disconnected) 3-manifold $M \subseteq C_{N}$ homeomorphic to $M^{\prime \prime}$. We can apply Theorem 7.2 to $M$ to obtain

$$
\operatorname{Vol}(M) \geq \frac{1}{2} V_{3}\|D M\|
$$

If $M$ is not a proper submanifold of $C_{N}$, then $\partial C_{N}$ must be a minimal surface to begin with. This is possible only if $\partial C_{N}$ is totally geodesic. Therefore if $C_{N}$ is not totally geodesic, then $M$ is a proper submanifold of the convex core, yielding the inequality

$$
\operatorname{Vol}\left(C_{N}\right)>\operatorname{Vol}(M) \geq \frac{1}{2} V_{3}\left\|D C_{N}\right\| .
$$

Proving the same inequality in the presence of cusps presents only technical difficulties. The first is determining the proper statement of the theorem. This we will attempt without introducing the language of pared 3-manifolds. (For a more detailed discussion of the cusped case see [42.) Let $N$ be a complete geometrically finite hyperbolic 3-manifold without boundary. Let $C_{N}$ be its convex core. Assume that $C_{N}$ is not compact. Then $D C_{N}$ is topologically a 3 -manifold with ends homeomorphic to a torus cross a ray. (See [30] for a precise description of the ends of $C_{N}$.) Cut off the ends at an embedded $\pi_{1}$-injective torus to form a compact 3-manifold with torus boundary. Let us denote this compact manifold by $\overline{D C}_{N}$. The Gromov 
norm rel boundary of a compact 3-manifold with toroidal boundary is well defined [43. Ch. 6]. Abusing notation slightly, let us denote this relative Gromov norm also by $\left\|\overline{D C}_{N}\right\|$. (We will have no need of the nonrelative Gromov norm for manifolds with boundary. As one expects, $\left\|\overline{D C}_{N}\right\|$ equals half the Gromov norm of the closed manifold obtained by doubling $\overline{D C}_{N}$ along its boundary [43, Ch. 6], [41.) Using the product structure on $N-C_{N}\left[12\right.$ and the precise manner in which $C_{N}$ exits the cusps of $N$ [30], it can be shown that $\left\|\overline{D C}_{N}\right\|$ is an invariant of the relative homotopy type of $(N$, cusps of $N)$. (This follows from [24. See [42].)

Theorem 8.2. Let $N$ be a complete geometrically finite hyperbolic 3-manifold without boundary. Assume that the convex core $C_{N}$ is not compact. Let $\overline{D C}_{N}$ be the truncated double of $C_{N}$. Then

$$
\operatorname{Vol}\left(C_{N}\right) \geq \frac{1}{2} V_{3}\left\|\overline{D C}_{N}\right\|
$$

Moreover, if $2 \operatorname{Vol}\left(C_{N}\right)=V_{3}\left\|\overline{D C}_{N}\right\|$, then $C_{N}$ has totally geodesic boundary.

Note that if $N$ is geometrically infinite, then by definition $\operatorname{Vol}\left(C_{N}\right)=\infty$, so geometric finiteness is not a restrictive hypothesis.

Proof. Let us assume that the boundary of $C_{N}$ is not totally geodesic. We will now describe a process for producing minimal surfaces in noncompact convex cores. A similar procedure is described in [20, Thm. 4.4].

Take an exhausting sequence of compact submanifolds $\left\{N_{i}\right\}$ of $N$ each of whose boundary is a collection of horospherical annuli and/or tori, so that the boundary of $N_{i+1}$ is distance at least $D_{i+1}$ from the boundary of $N_{i}$, where $\left\{D_{i}\right\}$ is a sequence we specify next.

By picking $D_{i}$ sufficiently large, the Riemannian metric on each $N_{i}$ can be altered near its boundary to produce a manifold $N_{i}^{\prime}$ with the following properties:

1. The metric is unchanged on $N_{i-1} \subset N_{i}^{\prime}$.

2. A collar of the boundary of $N_{i}^{\prime}$ has a flat (i.e. zero curvature) product metric with totally geodesic annular or toroidal boundary.

3. The sectional curvature of $N_{i}^{\prime}$ is between $-1-\varepsilon_{i}$ and zero, where $\varepsilon_{i} \rightarrow 0$.

4. The volume of $\left(N_{i}^{\prime} \cap C_{N}\right)-\left(N_{i-1} \cap C_{N}\right)$ goes to zero.

5. The submanifold $N_{i}^{\prime}-N_{i-1}$ has symmetry group given by isometries of the boundary annulus $\partial N_{i-1}$.

For the construction of such a metric see [27, 5]. (In Proposition 2.3 of [27] and in [5] an analogous construction is done in the finite volume setting. Here, the geometry of the cusp is unchanged, and the topology is simpler.)

By Dehn's lemma we can compress a finite number of homotopically trivial curves in $\partial C_{N}$ to obtain a $\pi_{1}$-injective embedded surface $S^{\prime \prime} \subset C_{N}$. Throw away components of $S^{\prime \prime}$ bounding $I$-bundle regions to get a surface $S^{\prime}$. Double each manifold $N_{i}^{\prime}$ along its boundary to produce a smooth Riemannian manifold $Z_{i}$. The surface $S^{\prime} \subset C_{N}$ doubles in $Z_{i}$ to a closed surface $\Sigma_{i}^{\prime} \subset Z_{i}$. There is a compact convex submanifold $C_{Z_{i}}$ in each $Z_{i}$ consisting of the points originally coming from the two copies of the convex core of $N$ (the convexity follows from the convexity of $C_{N}$ and the symmetry assumption 5). In each $Z_{i}$, there is a distance decreasing map on the complement of $C_{Z_{i}}$ defined by projecting to the nearest point of $\partial C_{Z_{i}}$. Using this distance-decreasing map to push wayward surfaces back into $C_{Z_{i}}$, one can produce an embedded area-minimizing surface $\Sigma_{i} \subset C_{Z_{i}}$ isotopic to $\Sigma_{i}^{\prime}$ [13, 21] 
(the surfaces cannot degenerate to an immersion, since we have thrown away all components of $S^{\prime \prime}$ bounding $I$-bundles). Consider the intersection $S_{i}$ of the surface $\Sigma_{i}$ with the hyperbolic submanifold $N_{i-1}$ of $N_{i}^{\prime}$. Here $S_{i}$ is a stable minimal surface rel boundary inside $N_{i-1}$, since $\Sigma_{i}$ is stable. Therefore its curvature is less than or equal to -1 at interior points.

Consider now the sequence of surfaces $S_{j} \subset N_{i}$ for $j>i$. By the Gauss-Bonnet theorem these surfaces have uniformly bounded area. Also, the fact that they are stable minimal surfaces implies that for each $i$ their principal curvatures are uniformly bounded in $N_{i}$ [37. They therefore form a precompact family. Take $i$ to infinity and use a diagonalization argument to obtain a limit $S \subset C_{N}$ which is also a smooth minimal immersion 21 into the original manifold $N$. Since the original surface $S^{\prime}$ was embedded, it follows that the limit surface $S$ is also embedded [13].

The surface $S$ is isotopic to $S^{\prime}$ (this is not hard to show, given the controlled geometry of $S$ ), so $S$ bounds a hyperbolic 3-manifold $X \subset C_{N}$. Recall that we assumed that $\partial C_{N}$ is not totally geodesic. It follows that $S$ is not equal to $\partial C_{N}$, so $\operatorname{Vol}\left(C_{N}\right)>\operatorname{Vol}(X)$. Let $X_{i} \subset C_{Z_{i}}$ be the submanifold bounded by $\Sigma_{i}$. Using the fact that the volume of $\left(N_{i}^{\prime} \cap C_{N}\right)-\left(N_{i-1} \cap C_{N}\right)$ goes to zero, one can show that the volume of $X_{i}$ converges to twice the volume of $X$. We may therefore pick an $i_{0}$ sufficiently large so that

$$
2 \operatorname{Vol}\left(C_{N}\right)>\left(1+\varepsilon_{i_{0}}\right)^{3 / 2} \operatorname{Vol}\left(X_{i_{0}}\right) .
$$

Here $-1-\varepsilon_{i_{0}}$ is the lower curvature bound for $N_{i}^{\prime}$ from condition 3 above. Define $c:=\sqrt{1+\varepsilon_{i_{0}}}$ and let $c X_{i_{0}}$ denote the space $X_{i_{0}}$ homothetically expanded by the factor $c$. The scaled space $c X_{i_{0}}$ has curvature bounded below by -1 .

Now apply Theorem 7.2 to $c X_{i_{0}}$. This yields the inequality

$$
\left(1+\varepsilon_{i_{0}}\right)^{3 / 2} \operatorname{Vol}\left(X_{i_{0}}\right)=\operatorname{Vol}\left(c X_{i_{0}}\right) \geq \frac{1}{2} V_{3}\left\|D X_{i_{0}}\right\|
$$

It remains only to prove that the Gromov norm of $D X_{i_{0}}$ is twice that of $\overline{D C}_{N}$ (rel its boundary). Starting with $\overline{D C}_{N}$, we can cut along a finite collection of embedded essential spheres, fill in the new boundary with balls, throw away $I$-bundle components, and then double the resulting manifold along its toroidal boundary to obtain a manifold homeomorphic to $D X_{i_{0}}$. Cutting along essential spheres or $S^{1}$ bundles does not alter the Gromov norm, and the Gromov norm adds when gluing two manifolds along essential boundary tori [43, Ch. 6], 41]. Therefore these operations going from $\overline{D C}_{N}$ to $D X_{i_{0}}$ double the Gromov norm.

\section{Noncompact CASE}

We would like to extend Theorem 7.2 to the case of noncompact manifolds with minimal surface boundary. Unfortunately, we can only extend this theorem to a particular case.

Theorem 9.1. Let $\bar{N}$ be a compact manifold with interior $(N, g)$, a hyperbolic 3-manifold of finite volume. Let $\bar{\Sigma}$ be an embedded incompressible ( $\pi_{1}$-injective) surface in $\bar{N}$. Then

$$
\operatorname{Vol}(N, g) \geq \frac{1}{2} V_{3}\|D(N \backslash \backslash \Sigma)\| \geq-V_{8} \chi(G u t s(M \backslash \backslash \Sigma)) .
$$

Proof. We may proceed as in the proof of Theorem 8.2 and produce a sequence of manifolds $\left(Z_{i}, g_{i}\right)$ homeomorphic to $D(\bar{N})$ with $R\left(g_{i}\right) \geq-6$ and such that $D(\bar{\Sigma})$ is 
a minimal surface in $Z_{i}$. Then $\operatorname{Vol}\left(Z_{i} \backslash \backslash D(\bar{\Sigma})\right) \geq\|D(N \backslash \backslash \Sigma)\|$ by Theorem 7.2. and $\operatorname{Vol}\left(Z_{i}\right) \rightarrow 2 \operatorname{Vol}(N)$, so that the first inequality of the theorem follows.

The second inequality comes from the fact that for a manifold, $\frac{1}{2} V_{3}\|D(N \backslash \backslash \Sigma)\|=$ $\operatorname{Vol}(\operatorname{Guts}(N \backslash \backslash \Sigma))$ [43, Thm. 6.5.5], and from Miyamoto's inequality (see section 2) [29].

\section{LOWER BOUND ON MINIMAL VOLUME OF ORIENTABLE HYPERBOLIC 3-MANIFOLDS}

The following theorem was proven in previous unpublished work of Agol and Dunfield via a direct smoothing argument, improving on the main result of [2]. Now we may apply Proposition 4.2 to reprove this.

Theorem 10.1 (Agol-Dunfield). Let $M$ be a hyperbolic 3-manifold with metric $\nu$. Let $\gamma \subset M$ be a geodesic link in $M$ of length $l$ with an embedded open tubular neighborhood $C$ of radius $R$ and with complete hyperbolic metric $\tau$ on $M_{\gamma}=M-\gamma$. Then

$$
\operatorname{Vol}\left(M_{\gamma}, \tau\right) \leq(\operatorname{coth} 2 R)^{3}\left(\operatorname{Vol}(M, \nu)+\frac{\pi}{2} l \tanh R \tanh 2 R\right) .
$$

Proof. We follow the method of [2]. We have a tubular neighborhood $C$ about $\gamma$ of radius $R$. The mean curvature of $\partial C$ is $\kappa=\frac{1}{2}(\operatorname{coth} R+\tanh R)=\operatorname{coth} 2 R>1$. Remove $C$, and insert a cusp with curvature scaled so that the mean curvature on the boundary matches that of $\partial C$. Then we obtain a $C^{0}$ metric on $M_{\gamma}$ satisfying equation (2). Since a horotorus has mean curvature 1, we rescale the hyperbolic metric on the cusp to have sectional curvature $-\kappa^{2}$, by scaling lengths in the standard hyperbolic metric by a factor of $\kappa^{-1}$, which makes the horotori have mean curvature $\kappa$. The tube $C$ has volume $\pi l \sinh ^{2} R$, and the area of $\partial C=\pi l \sinh 2 R$. The cusp in the standard hyperbolic metric has volume half the area of its boundary horotorus, so a cusp with boundary of mean curvature $\kappa$ will have volume $\frac{1}{2 \kappa}$ times the area of its boundary. Thus, the volume of the cusp will be $\frac{\pi l \sinh 2 R}{2 \kappa}=\frac{1}{2} \pi l \sinh 2 R \tanh 2 R$. We must rescale by $\kappa$ in order that the minimal scalar curvature is -6 , obtaining a complete $C^{0}$ metric $h$ on $M_{\gamma}$ satisfying the hypotheses of Proposition 4.2, Then by Theorem 7.1 and by the above computations we have

$$
\begin{gathered}
\operatorname{Vol}\left(M_{\gamma}, \tau\right) \leq \operatorname{Vol}\left(M_{\gamma}, h\right)=\kappa^{3}\left(\operatorname{Vol}(M, \nu)-\pi l \sinh ^{2} R+\frac{1}{2} \pi l \sinh 2 R \tanh 2 R\right) \\
=\kappa^{3}\left(\operatorname{Vol}(M, \nu)+\pi l \sinh ^{2} R \operatorname{sech} 2 R\right) .
\end{gathered}
$$

To simplify things, we were slightly inaccurate in the argument in the previous paragraph, since Theorem 7.1 only applies to closed manifolds. To remedy this, we perform high order Dehn filling on $M_{\gamma}$, by removing $C$ and inserting a tubular neighborhood of a geodesic in $\mathbb{H}^{3}$ with the boundary slope along a sequence of meridians $\alpha_{i}, i \rightarrow \infty$. As the length of the meridian $\alpha_{i}$ goes to $\infty$, the inserted tube will converge in the Gromov-Hausdorff limit to the horotorus described in the previous paragraph, and we obtain the same estimate by taking a limit of estimates obtained using these Dehn filled metrics. (Alternatively, one may use the doubling trick of Theorem 8.2 )

The following result improves on Proposition 5.4 of [35].

Corollary 10.2. The minimal volume orientable hyperbolic 3-manifold $M$ has $\operatorname{Vol}(M) \geq 0.67$. 
Proof. This follows as in the proof of Proposition 5.4 of [35, using Theorem 10.1 to replace Theorem 5.2 of [35]. Let $M$ be a minimal volume orientable hyperbolic 3 -manifold. Let $\gamma$ be a minimal length closed geodesic in $M$ of length $l$. Since $M$ is minimal volume, $\gamma$ has a tube about it of radius $R \geq \log (3) / 2$ (see [16, Cor. 4.3]). By the tube packing argument of [35, Cor. 4.4], the volume of the tube around $\gamma$ satisfies $\pi \sinh ^{2}(R) \geq .91 \mathrm{Vol}(M)$. Thus, from Theorem 10.1, we see that $\operatorname{Vol}\left(M_{\gamma}, \tau\right) \leq \operatorname{coth}^{3}(2 R)(1+.91 \operatorname{sech}(2 R)) \operatorname{Vol}(M)$. This function is increasing with $R$, and $\operatorname{Vol}\left(M_{\gamma}, \tau\right) \geq 2.02988$ by [9, so plugging $R=\log (3) / 2$ into the above inequality, we see that $\operatorname{Vol}(M) \geq .67$.

Remark. Theorem 10.1 allows us to assail the problem of finding the minimal volume hyperbolic 3-manifold on various fronts, by either classifying cusped orientable hyperbolic 3 -manifolds with volume $<2.852$ (for example, by extending the arguments of Cao and Meyerhoff [9]) or by classifying closed 3-manifolds with a minimal length geodesic with tube radius $<0.7$.

\section{UNIVERSAL MANIFOLD PAIRINGS}

The paper 14 defines the notion of a universal $(n+1)$-manifold pairing. This consists of linear combinations of $(n+1)$-manifolds with marked boundary, together with a pairing on these manifolds which have the same $n$-dimensional marked boundary which takes values in formal linear combinations of closed $(n+1)$-manifolds. A unitary TQFT may be thought of as a "representation" of such a pairing, assigning a Hermitian vector space to each closed $n$-manifold and a number to each closed $(n+1)$-manifold and satisfying some extra axioms 3]. Conjecture 2.2 of 14 states that the $(2+1)$-dimensional universal manifold pairing faithfully detects linear combinations of 3-manifolds (there are no Hermitian isotropic vectors). We provide some partial evidence for this conjecture in a special case.

Let $\Sigma$ be a closed oriented surface, and let $\mathcal{M}_{\Sigma}$ denote the formal combinations of compact oriented 3-manifolds $M$ with $\partial M=\Sigma$ and coefficients in $\mathbb{C}$. Define the pairing $():, \mathcal{M}_{\Sigma} \times \mathcal{M}_{\Sigma} \rightarrow \mathcal{M}$ by $\left(\sum_{i} a_{i} M_{i}, \sum_{j} b_{j} N_{j}\right)=\sum_{i, j} a_{i} \bar{b}_{j} M_{i} \cup_{\Sigma} \bar{N}_{j}$ (where $\mathcal{M}=\mathcal{M}_{\emptyset}$ and $\bar{N}_{j}$ denotes $N_{j}$ with the reversed orientation). The following conjecture generalizes in a natural way Conjecture 2.2 of [14].

Conjecture 11.1. If there exists $x_{1}, \ldots, x_{m} \in \mathcal{M}_{\Sigma}$ such that $\sum_{i}\left(x_{i}, x_{i}\right)=0 \in \mathcal{M}$, then $x_{1}=\cdots=x_{m}=0$.

We provide some evidence for this conjecture in a special case.

Corollary 11.2. Suppose that each $x_{1}, \ldots, x_{m} \in \mathcal{M}_{\Sigma}$ is a formal linear combination of compact, acylindrical 3-manifolds, such that $\sum_{i}\left(x_{i}, x_{i}\right)=0 \in \mathcal{M}$. Let $x_{i}=\sum_{j \in I_{i}} x_{i j} M_{j}$, and suppose that $\sum_{i}\left|I_{i}\right|^{2}$ is minimal amongst $x_{i}$ such that $\sum_{i}\left(x_{i}, x_{i}\right)=0$. Then for $j, k \in I_{i},\left\|M_{j} \cup_{\Sigma} \bar{M}_{k}\right\|=\left\|M_{j} \cup_{\Sigma} \bar{M}_{j}\right\|$.

Proof. Let $g_{j}$ denote the hyperbolic metric on $M_{j}$ with totally geodesic boundary. Denote the manifold $M_{j k}=M_{j} \cup_{\Sigma} \bar{M}_{k}, j, k \in I_{i}$. Let $\Sigma_{j k} \subset M_{j k}$ be a least area representative of $\Sigma \subset M_{j k}$ in the canonical hyperbolic metric on $M_{j k}$. Then $\left\|M_{j k}\right\| \geq \frac{1}{2}\left(\left\|M_{j j}\right\|+\left\|M_{k k}\right\|\right)$ by Theorem 7.2 . Since $\sum_{i, j}\left|x_{i j}\right|^{2} M_{j j} \neq 0$, in order for $\sum_{i}\left(x_{i}, x_{i}\right)=0$, we must have $M_{a a}=M_{b c}$, for some $b \neq c, b, c \in I_{i}$. Moreover, we may assume that $\left\|M_{a a}\right\|$ is minimal over $\left\|M_{j j}\right\|, j \in \bigcup_{i} I_{i}$. Thus, $\left\|M_{a a}\right\|=$ $\left\|M_{b c}\right\| \geq \frac{1}{2}\left(\left\|M_{b b}\right\|+\left\|M_{c c}\right\|\right) \geq\left\|M_{a a}\right\|$ (the second inequality follows from the 
assumed minimality of $\left.\left\|M_{a a}\right\|\right)$, and thus $\left\|M_{a a}\right\|=\left\|M_{b b}\right\|=\left\|M_{c c}\right\|=\left\|M_{b c}\right\|$. Since we have equality, we must have that $\Sigma_{b c}$ is totally geodesic in $M_{b c}$ by Theorem 7.2, and thus $g_{b}$ and $g_{c}$ induce the same metric up to isotopy on $\Sigma$. Take the maximal subpartition $J=\left\{J_{1}, \ldots, J_{n}\right\}$ of $I=\left\{I_{1}, \ldots, I_{m}\right\}$ such that for all $i, j \in J_{l}$, $\left\|M_{i j}\right\|=\left\|M_{a a}\right\|$. This partition is a subset of the partition by the isotopy class of the metric on $\Sigma=\partial M_{j}$ induced by $g_{j}$, and therefore it is easy to see that $\bigcup J$ includes all $j$ such that $\left\|M_{j j}\right\|=\left\|M_{a a}\right\|$. Then for $i$ not in $\bigcup J, i \neq j,\left\|M_{i j}\right\|>\left\|M_{a a}\right\|$, and thus all the cancellation among the terms of $\sum_{i}\left(x_{i}, x_{i}\right)$ with $M_{a a}$ must occur among the terms of the partition $J$. Associated to $J_{l}$, there is a vector $y_{l}$, where $y_{l}=\sum_{j \in J_{l}} x_{i j} M_{j}$, where $J_{l} \subset I_{i}$. Then $\sum_{l}\left(y_{l}, y_{l}\right)=0$, and $\sum_{l}\left|J_{l}\right|^{2} \leq \sum_{i}\left|I_{i}\right|^{2}$. By the minimality hypothesis on $I$, we have $J=I$, and the result follows.

This reduces this special case of Conjecture 2.2 of [14 to a geometric question about how many distinct ways a hyperbolic manifold may be obtained by gluing together two copies of a manifold with geodesic boundary by the identity on the boundary. It seems promising that this question may yield to geometric techniques.

\section{Conclusion}

The results in this paper give rise to many interesting questions. A natural question is whether one may prove the main result without using Ricci flow.

Conjecture 12.1. If a finite volume hyperbolic 3-manifold with minimal surface boundary is locally minimal among such manifolds, then the boundary is totally geodesic.

If this conjecture were true, then one should be able to reprove Theorem 7.2 by deforming a hyperbolic manifold with minimal surface boundary to have geodesic boundary, while decreasing volume.

Another possible approach would be to try to use the methods of the natural map [6]. If $(M, g)$ is a Riemannian metric, let $h(M, g)$ denote the volume entropy of $g$.

Conjecture 12.2. If $(M, g)$ is a closed Riemannian 3-manifold with $R(g) \geq-6$, then $h(M, g) \leq h\left(\mathbb{H}^{3}\right)$.

This would enable one to reprove the main theorem using the techniques of the natural map.

One nagging point we were unable to resolve is to prove the main theorem in the case that the manifold is noncompact.

Conjecture 12.3. Let $(M, g)$ be a complete finite volume 3-manifold with minimal surface boundary and scalar curvature $R(g) \geq-6$. Then $\operatorname{Vol}(M, g) \geq \frac{1}{2}\|D M\|$, with equality if and only if $M$ has geodesic boundary.

This conjecture might be useful in an attempt to prove Conjecture 2.2 of [14, by extending the arguments of Corollary 11.2 .

\section{Appendix A. Volume change under Drilling: theory Vs. EXPERIment}

Let $M$ be a closed hyperbolic 3-manifold, and let $\gamma$ be a simple geodesic in $M$. Consider the complement $M_{\gamma}=M \backslash \gamma$. This appendix focuses on the following question: how are the volumes of $M$ and $M_{\gamma}$ related? In general, Thurston showed that $\operatorname{Vol}(M)<\operatorname{Vol}\left(M_{\gamma}\right)$, so the goal here is to control the increase in volume when 
we drill out $\gamma$. To be able to do this, we need more information about the geometry of $M$, so assume in addition that we know the length $L$ of $\gamma$ and the radius $R$ of a maximal embedded open tube about $\gamma$. (Equivalently, $R$ is half the minimal distance between two distinct lifts of $\gamma$ to the universal cover of $M$.) To simplify the notation, set $V_{\text {fill }}=\operatorname{Vol}(M)$ and $V_{\text {drill }}=\operatorname{Vol}\left(M_{\gamma}\right)$. The volume estimates I will discuss involve the term

$$
B=V_{\text {fill }}+\pi L \sinh ^{2}(R) \operatorname{sech}(2 R) .
$$

In 2, Agol showed that

$$
V_{\text {drill }} \leq C_{O} B \quad \text { where } \quad C_{O}=(\operatorname{coth}(R) \operatorname{coth}(2 R))^{3 / 2} .
$$

Using Perelman's work, Agol and I improved this to

$$
V_{\text {drill }} \leq C_{P} B \quad \text { where } \quad C_{P}=\operatorname{coth}^{3}(2 R) .
$$

See Theorem 10.1 in the body of this paper for a proof. The following plot shows the ratio $C_{O} / C_{P}$ of the multiplicative factors as a function of $R$ :

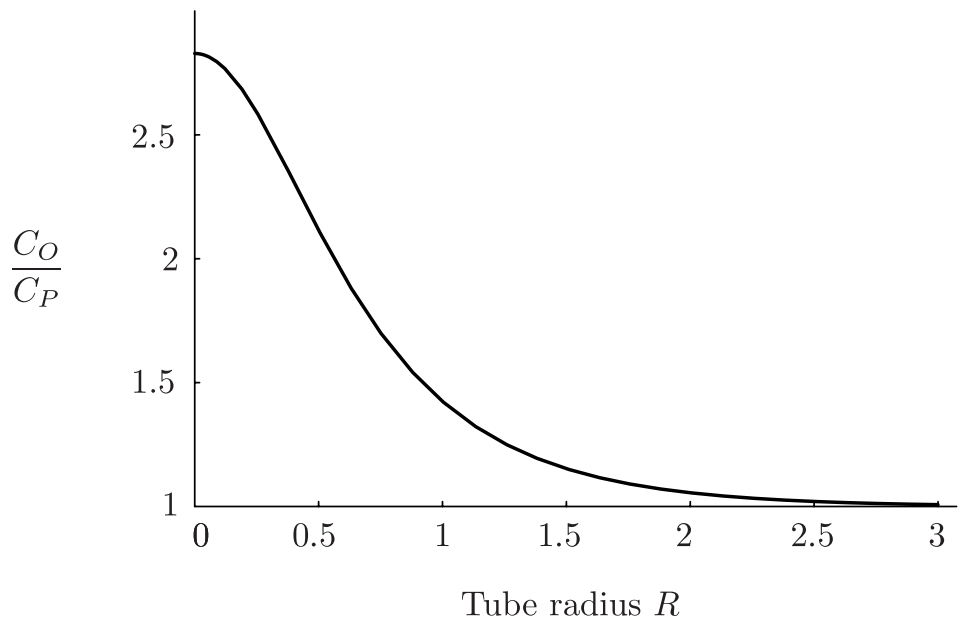

As you can see, the new estimate is markedly better than the old one for small $R$, but the two estimates are asymtopically the same for large $R$.

The purpose of this appendix is to compare the new drilling estimate with data from more than 25,000 pairs $(M, \gamma)$. In all cases, the data satisfies (7). Moreover, the estimate in (7) is fairly good for $R>1$. Since (7) rests on Perelman's announced proof of geometrization [34, these results can be viewed as an experimental check on the correctness of his proof. There are two obvious caveats here: only a finite number of manifolds were examined and only part of Perelman's work is needed (namely Theorem 3.1 in the body of this paper). There is a more subtle issue as well: it is entirely possible that for these examples, when one applies the proof of Theorem 3.1] the Ricci flow never goes through any surgeries. That is, these examples could well avoid one of the most subtle parts of Perelman's approach.

After briefly discussing the sample manifolds, I will give various graphical comparisons between (7) and the data. I will then turn to an alternate way of explaining the data, namely hyperbolic Dehn surgery. This approach more closely models the data than (7), but does not provide universal bounds as (7) does. 
A.1. The sample. The sample consists of 25,709 pairs $(M, \gamma)$, where the manifolds $M$ were drawn from the Hodgson-Weeks census [45. The geometry of selected geodesics was computed using Oliver Goodman's program Tube, which is distributed with his program Snap 17. The output of the program Tube is not completely rigorous, but it should be essentially correct for nearly all of the examples. Most of the geodesics are not too long $(L \leq 2.5)$ and have large tube radii $(R \geq 0.4)$. Concentrating on large tube radii was forced because Tube had difficulty with the longer geodesics. However, as you will see, the estimate (7) is really poor even for modestly small $R$, so this omission is of no real importance. While most of the geodesics are fairly short, to concentrate on the regime where the two estimates differ noticeably, I did require the geodesics to have length $L \geq 0.3$. In more than 24,000 of the cases, the manifold $M_{\gamma}$ has a triangulation with $\leq 7$ tetrahedra. Partially as a result of having such simple complements, I expect that, in nearly all cases, one can do hyperbolic Dehn filling from the drilled manifold to the closed one.

A.2. Experimental results. Let $(M, \gamma)$ be as usual. As per (7) set $V_{\text {est }}=C_{P} B$. Then we have

$$
V_{\text {fill }}<V_{\text {drill }} \leq V_{\text {est }} .
$$

To understand how good a bound $V_{\text {est }}$ is, we consider the overshoot error $V_{\text {est }}-V_{\text {drill }}$ as a proportion of the actual increase in volume $V_{\text {drill }}-V_{\text {fill }}$. That is, in Figure 1 we plot

$$
\frac{V_{\text {est }}-V_{\text {drill }}}{V_{\text {drill }}-V_{\text {fill }}}
$$

against the tube radius $R$. (One could just compare the error with $V_{\text {drill }}$, but that provides less information when the tube radius is large, in which case $V_{\text {drill }}$ is only

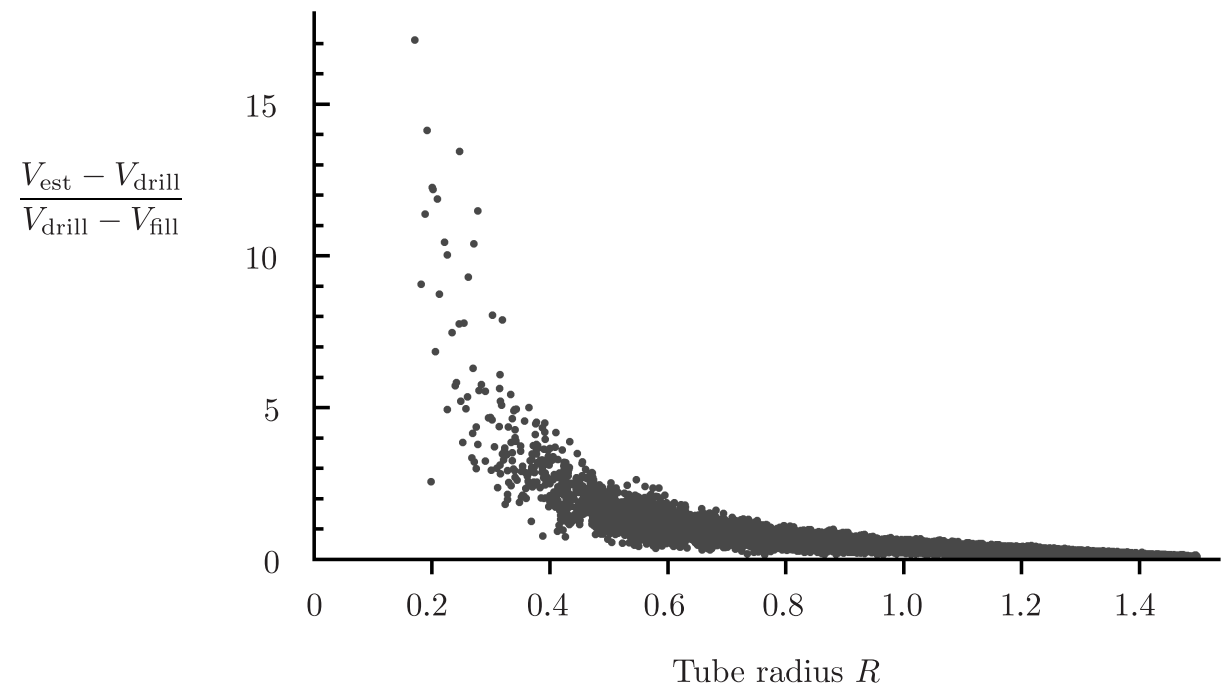

Figure 1. This plot shows the amount that (7) overshoots the actual volume $V_{\text {drill }}$, compared via (8). Three data points are omitted; in each case $R<0.15$ and the vertical coordinate is $>40$. 

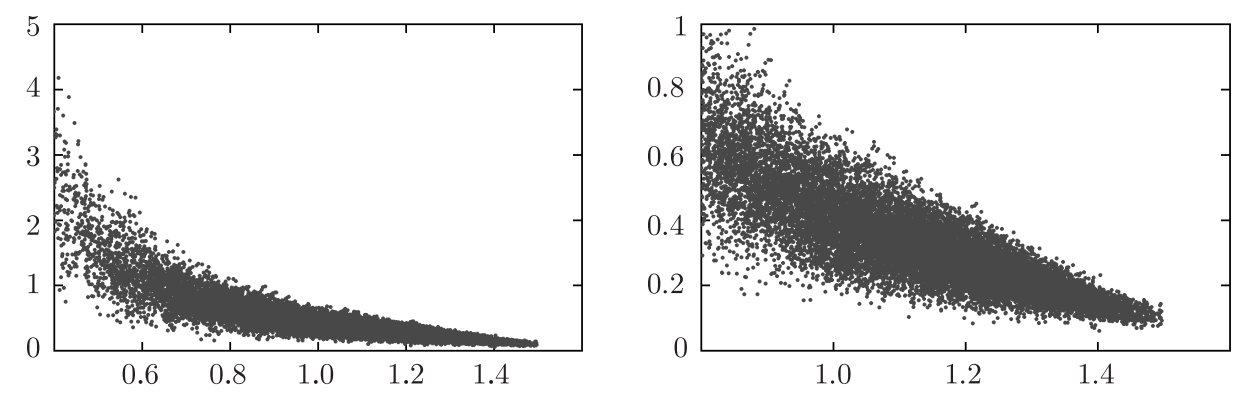

Figure 2. The data just for larger tube radii $R$. The horizontal axis remains $R$, and the vertical axis the quantity (8).

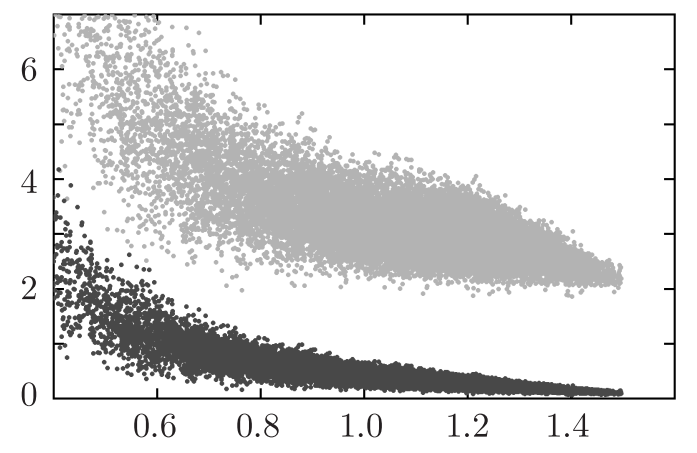

Figure 3. The top band is the estimate using the factor $C_{O}$, compared to the improved version using $C_{P}$ (bottom band). As before, the horizontal axis remains the tube radius $R$, and the vertical axis the quantity (8).

a very small amount higher than $V_{\text {fill }}$.) Note that the vertical scale is quite large - for the small $R$, the error quantity (8) is more than 15 . Thus for small $R$, the estimate is quite poor. This is not so surprising - it makes intuitive sense that the metric constructed in the proof of (7) is far (in any reasonable sense) from the hyperbolic metric on $M_{\gamma}$ when $R$ is small. Thus one must expect a larger drop in the normalized volume $V_{R}\left(M_{\gamma}\right)$ during the Ricci flow with surgery, making the gap in Theorem 3.1 larger. Now, let us look at closeups for larger $R$, where the estimate is markedly better; these are shown in Figure 2. It is also worth comparing Agol's original estimate to the Perelman enhanced estimate; this is done in Figure 3 .

Another way to compare the data with the estimate is this. Both of the bounds have the same form $V_{\text {drill }} \leq C(R) B$. It is natural to ask: experimentally, what is the optimal form of the function $C(R)$ in such an estimate? Equivalently, we seek the function that just barely satisfies

$$
\frac{1}{C(R)} \leq \frac{B}{V_{\text {drill }}}
$$

Figures 45 show the left-hand side of the above, plotted against $1 / C_{P}$ and $1 / C_{O}$.

One intriguing thing here is that $B / V_{\text {drill }}$ is always $\leq 1$. In other words,

$$
V_{\text {fill }}+\pi L \sinh ^{2}(R) \operatorname{sech}(2 R) \leq V_{\text {drill }}
$$




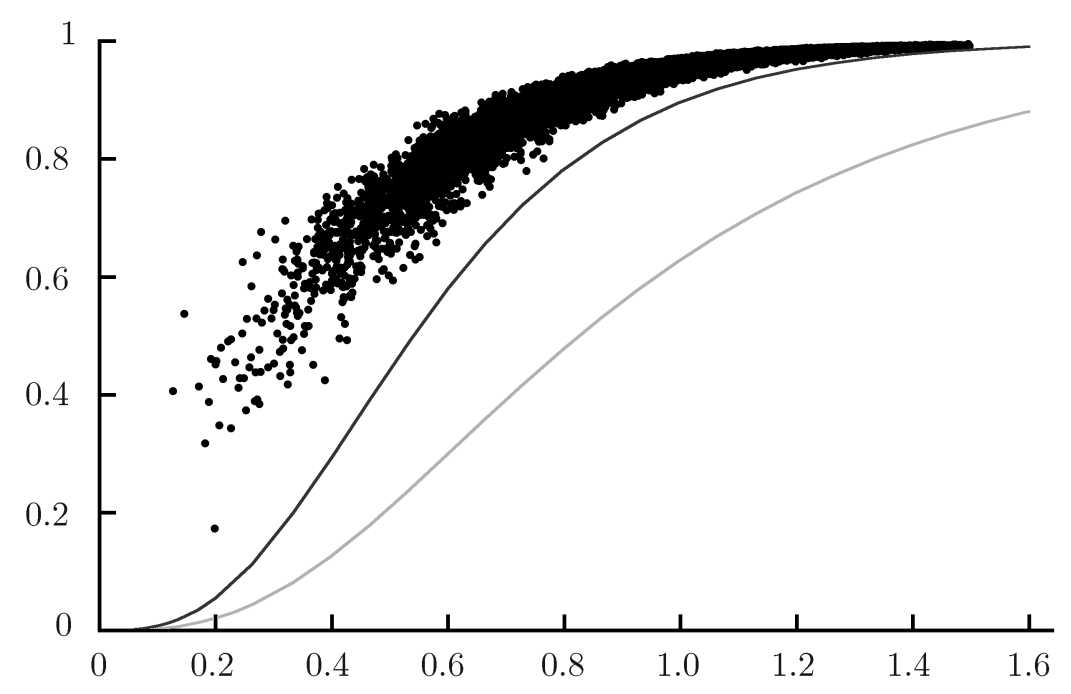

Tube radius $R$

Figure 4. Here, the vertical axis for the data is $B / V_{\text {drill. }}$. For (7) to hold, the data points must lie above $1 / C_{P}(R)$ which is the upper curve. The lower curve is $1 / C_{O}(R)$.
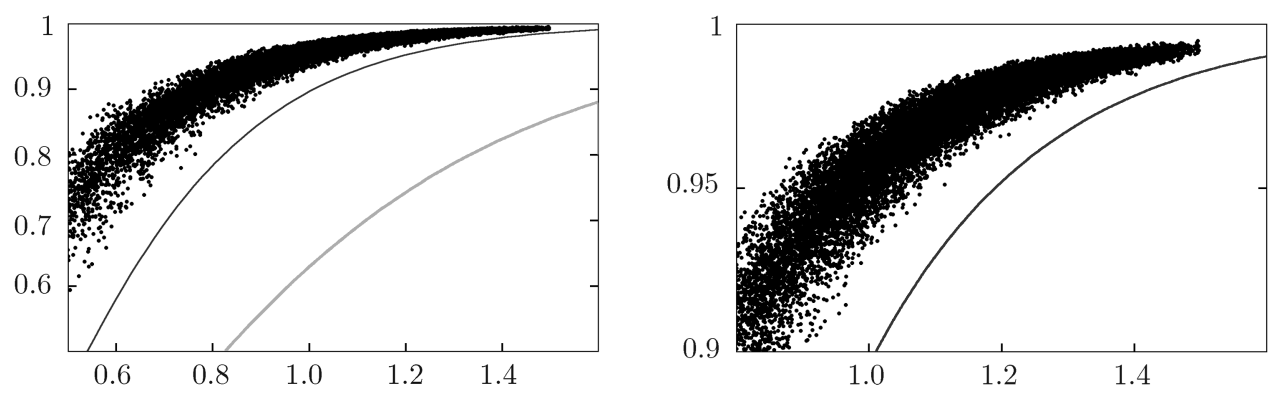

Figure 5. Closeups of the data in Figure 4.

This is not predicted by Theorem 10.1, and I do not see any reason why it should hold true in general. However, I also know of no counterexamples.

A.3. Hyperbolic Dehn surgery and volume change. If the hyperbolic metric on $M$ can be deformed to the hyperbolic metric on $M_{\gamma}$ through a series of cone manifolds with cone locus $\gamma$, we say that $M$ is obtained from $M_{\gamma}$ by hyperbolic Dehn filling. In this case, Schläfli's formula [4] gives that

$$
\Delta V=V_{\text {drill }}-V_{\text {fill }}=\frac{1}{2} \int_{0}^{2 \pi} L(\theta) d \theta
$$

where $L(\theta)$ is the length of $\gamma$ in the metric with cone angle $\theta$.

If we consider different hyperbolic Dehn fillings on a fixed cusp manifold, Neumann and Zagier 32 showed that $\Delta V \sim \pi L / 2$ as the length $L$ of the core geodesic goes to 0 . For a particular Dehn filling, Bridgeman [8] observed that as long as $L(\theta)$ 
is an increasing function of $\theta$, then (9) implies that the volume increase is bounded by twice the asymptotic estimate:

$$
\Delta V \leq \pi L
$$

While it appears that Bridgeman's inequality does not hold in general [2, Sec. 3], Hodgson and Kerckhoff showed that it does hold when $L \leq 0.16$, provided also that the tube radius $R \geq 0.66$ [22, Sec. 6]. In the same paper, Hodgson and Kerckhoff gave a very precise version of the asymptotics of $\Delta V \sim \pi L / 2$ with similar hypotheses. Given these results, it makes sense to plot $\Delta V /(\pi L)$ as a function of $L$ :

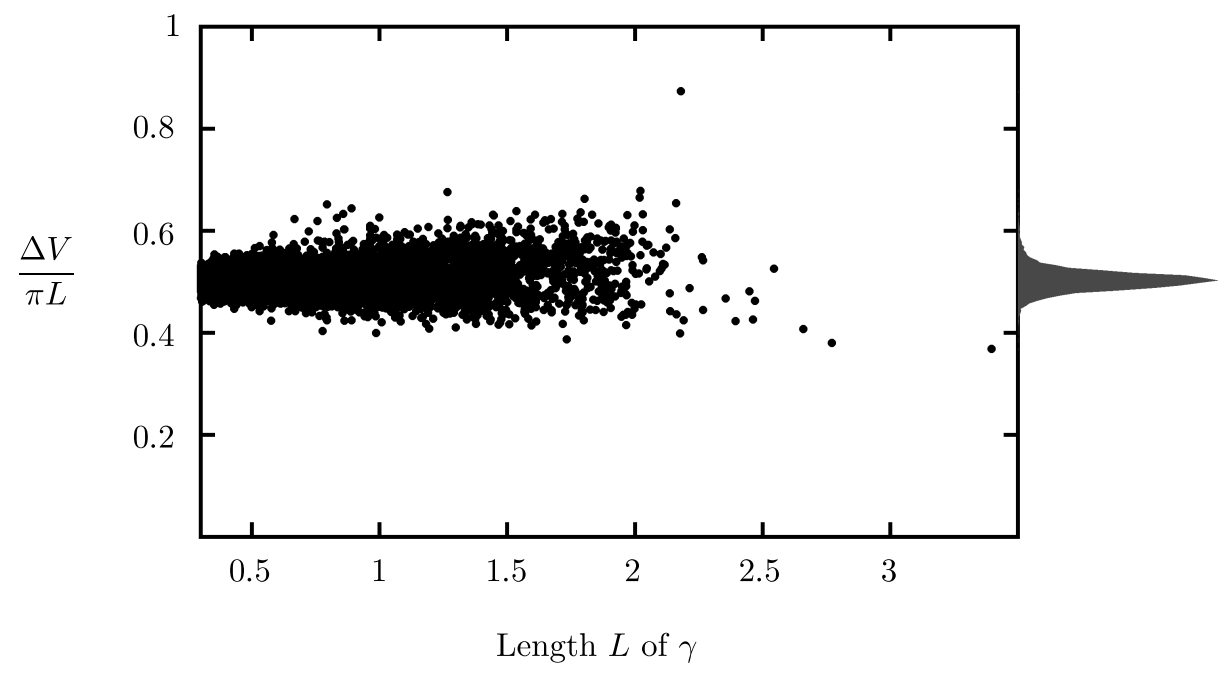

As you can see, the result is the simplest picture we have seen yet - the distribution of $\Delta V /(\pi L)$ is nearly independent of $L$, with some broadening as we increase $L$. The histogram attached to the right side of the plot gives the distribution of $\Delta V /(\pi L)$, which is a bell-curve shape with mean 0.5034 (essentially the $1 / 2$ expected!) and standard deviation $\sigma=0.022$. Indeed, the distribution is very nearly the same as the normal distribution with mean 0.502 and $\sigma=0.017$; the main difference is that the top tail is a little thicker than the normal distribution. In all cases, Bridgeman's inequality (10) holds.

While the picture coming from hyperbolic Dehn filling provides the most accurate fit for our data, I should emphasize that it is not known whether one can always obtain $M$ from $M_{\gamma}$ via hyperbolic Dehn filling. Even when this is known, one can only estimate $\Delta V$ if one makes additional assumptions about the behavior of $L(\theta)$. The estimate (7) has the advantage that it applies without any additional hypotheses, which is important in applications such as bounding the volume of the smallest closed hyperbolic 3-manifold.

\section{ACKNOWLEDGEMENTS}

We thank Pengzi Miao, Andre Neves, and Rick Schoen for helpful correspondence. We also thank the referee for many helpful suggestions. 


\section{REFERENCES}

[1] Ian Agol, Lower bounds on volumes of hyperbolic Haken 3-manifolds, arXiv:math.GT/ 9906182.

[2] C Volume change under drilling, Geom. Topol. 6 (2002), 905-916 (electronic). MR.1943385 (2004e:57021)

[3] Michael Atiyah, Quantum field theory and low-dimensional geometry, Progr. Theoret. Phys. Suppl. (1990), no. 102, 1-13 (1991), Common trends in mathematics and quantum field theories (Kyoto, 1990). MR.1182158 (95f:57050)

[4] Thierry Aubin, Some nonlinear problems in Riemannian geometry, Springer Monographs in Mathematics, Springer-Verlag, Berlin, 1998. MR1636569(99i:58001)

[5] Laurent Bessières, Sur le volume minimal des variétés ouvertes, Ann. Inst. Fourier (Grenoble) 50 (2000), no. 3, 965-980. MR.1779901 (2001g:53064)

[6] Gérard Besson, Gilles Courtois, and Sylvestre Gallot, Lemme de Schwarz réel et applications géométriques, Acta Math. 183 (1999), no. 2, 145-169. MR.1738042 (2001c:53038)

[7] Hubert L. Bray, Proof of the Riemannian Penrose inequality using the positive mass theorem, J. Differential Geom. 59 (2001), no. 2, 177-267. MR1908823 (2004j:53046)

[8] Martin Bridgeman, Bounds on volume increase under Dehn drilling operations, Proc. London Math. Soc. (3) 77 (1998), no. 2, 415-436. MR1635161 (99f:57019)

[9] Chun Cao and G. Robert Meyerhoff, The orientable cusped hyperbolic 3-manifolds of minimum volume, Invent. Math. 146 (2001), no. 3, 451-478. MR.1869847(2002i:57016)

[10] Huai-Dong Cao and Xi-Ping Zhu, A complete proof of the Poincaré and geometrization conjectures - application of the Hamilton-Perelman theory of the Ricci flow, Asian J. Math. 10 (2006), no. 2, 165-492. MR2233789

[11] Marc Culler and Peter B. Shalen, Volumes of hyperbolic Haken manifolds. I., Inventiones Mathematicae 118 (1994), no. 2, 285-329. MR1292114 (95g:57023)

[12] D. B. A. Epstein and A. Marden, Convex hulls in hyperbolic space, a theorem of Sullivan, and measured pleated surfaces, Analytical and geometric aspects of hyperbolic space (Coventry/Durham, 1984), London Math. Soc. Lecture Note Ser., vol. 111, Cambridge Univ. Press, Cambridge, 1987, pp. 113-253. MR903852 (89c:52014)

[13] Michael Freedman, Joel Hass, and Peter Scott, Least area incompressible surfaces in 3manifolds, Invent. Math. 71 (1983), no. 3, 609-642. MR695910(85e:57012)

[14] Michael H. Freedman, Alexei Kitaev, Chetan Nayak, Johannes K. Slingerland, Kevin Walker, and Zhenghan Wang, Universal manifold pairings and positivity, Geom. Topol. 9 (2005), 2303-2317 (electronic). MR 2209373 (2006k:57080)

[15] David Gabai and William H. Kazez, Group negative curvature for 3-manifolds with genuine laminations, Geom. Topol. 2 (1998), 65-77 (electronic). MR.1619168(99e:57023)

[16] David Gabai, G. Robert Meyerhoff, and Nathaniel Thurston, Homotopy hyperbolic 3manifolds are hyperbolic, Ann. of Math. (2) 157 (2003), no. 2, 335-431. MR1973051 (2004d:57020)

[17] Oliver Goodman, Snap: a computer program for studying arithmetic invariants of hyperbolic 3-manifolds, www.ms.unimelb.edu.au/ snap.

[18] Richard S. Hamilton, Non-singular solutions of the Ricci flow on three-manifolds, Comm. Anal. Geom. 7 (1999), no. 4, 695-729. MR1714939 (2000g:53034)

[19] Joel Hass, Acylindrical surfaces in 3-manifolds, Michigan Mathematical Journal 42 (1995), no. 2, 357-365. MR.1342495 (96c:57031)

[20] Joel Hass, J. Hyam Rubinstein, and Shicheng Wang, Boundary slopes of immersed surfaces in 3-manifolds, J. Differential Geom. 52 (1999), no. 2, 303-325. MR1758298 (2001h:57023)

[21] Joel Hass and Peter Scott, The existence of least area surfaces in 3-manifolds, Trans. Amer. Math. Soc. 310 (1988), no. 1, 87-114. MR965747 (90c:53022)

[22] Craig D. Hodgson and Steven P. Kerckhoff, Universal bounds for hyperbolic Dehn surgery, Ann. of Math. (2) 162 (2005), no. 1, 367-421. MR2178964 (2006g:57031)

[23] William H. Jaco and Peter B. Shalen, Seifert fibered spaces in 3-manifolds, Mem. Amer. Math. Soc. 21 (1979), no. 220, viii+192. MR539411 (81c:57010)

[24] Klaus Johannson, Homotopy equivalences of 3-manifolds with boundaries, Lecture Notes in Mathematics, vol. 761, Springer, Berlin, 1979. MR.551744 (82c:57005)

[25] Bruce Kleiner and John Lott, Notes on Perelman's papers, arXiv:math.DG/0605667. 
[26] Marc Lackenby, The volume of hyperbolic alternating link complements, Proc. London Math. Soc. (3) 88 (2004), no. 1, 204-224, with an appendix by Ian Agol and Dylan Thurston. MR2018964 (2004i:57008)

[27] Bernhard Leeb, 3-manifolds with(out) metrics of nonpositive curvature, Invent. Math. 122 (1995), no. 2, 277-289. MR 1358977 (97g:57015)

[28] Pengzi Miao, Positive mass theorem on manifolds admitting corners along a hypersurface, Adv. Theor. Math. Phys. 6 (2002), no. 6, 1163-1182 (2003). MR1982695 (2005a:53065)

[29] Yosuke Miyamoto, Volumes of hyperbolic manifolds with geodesic boundary, Topology 33 (1994), no. 4, 613-629. MR1293303 (95h:57014)

[30] John W. Morgan, On Thurston's uniformization theorem for three-dimensional manifolds, The Smith conjecture (New York, 1979), Academic Press, Orlando, FL, 1984, pp. 37-125. MR.758464

[31] John W. Morgan and Gang Tian, Ricci flow and the poincaré conjecture, 2006, arXiv:math.DG/0607607, preprint.

[32] Walter D. Neumann and D. Zagier, Volumes of hyperbolic three-manifolds, Topology 24 (1985), 308-332. MR815482 (87j:57008)

[33] Grisha Perelman, Ricci flow with surgery on three-manifolds, arXiv:math.DG/0303109.

[34] _ The entropy formula for the Ricci flow and its geometric applications, arXiv: math.DG/0211159.

[35] Andrew Przeworski, A universal upper bound on density of tube packings in hyperbolic space, J. Differential Geom. 72 (2006), no. 1, 113-127. MR.2215457 (2007b:57030)

[36] Michael Reed and Barry Simon, Methods of modern mathematical physics. IV. Analysis of operators, Academic Press [Harcourt Brace Jovanovich Publishers], New York, 1978. MR.0493421 (58:12429c)

[37] Richard Schoen, Estimates for stable minimal surfaces in three-dimensional manifolds, Seminar on minimal submanifolds, Ann. of Math. Stud., vol. 103, Princeton Univ. Press, Princeton, NJ, 1983, pp. 111-126. MR795231 (86j:53094)

[38] Richard M. Schoen, Variational theory for the total scalar curvature functional for Riemannian metrics and related topics, Topics in calculus of variations (Montecatini Terme, 1987), Lecture Notes in Math., vol. 1365, Springer, Berlin, 1989, pp. 120-154. MR994021 (90g:58023)

[39] Takashi Shioya and Takao Yamaguchi, Volume collapsed three-manifolds with a lower curvature bound, Math. Ann. 333 (2005), no. 1, 131-155. MR2169831(2006j:53050)

[40] Miles Simon, Deformation of $C^{0}$ Riemannian metrics in the direction of their Ricci curvature, Comm. Anal. Geom. 10 (2002), no. 5, 1033-1074. MR1957662 (2003j:53107)

[41] Teruhiko Soma, The Gromov invariant of links, Invent. Math. 64 (1981), no. 3, 445-454. MR632984 (83a:57014)

[42] Peter A. Storm, Hyperbolic convex cores and simplicial volume, arXiv:math.GT/0409312.

[43] William P. Thurston, The geometry and topology of 3-manifolds, Lecture notes from Princeton University, 1978-80.

[44] È. B. Vinberg, Volumes of non-Euclidean polyhedra, Uspekhi Mat. Nauk 48 (1993), no. 2(290), 17-46. MR 1239859 (94h:52012)

[45] Jeffery Weeks, SnapPea: A computer program for creating and studying hyperbolic 3manifolds, www.geometrygames.org.

Department of Mathematics, Computer Science, and Statistics, University of Illinois at Chicago, 322 SEO, m/c 249, 851 S. Morgan St., Chicago, Illinois 60607-7045

E-mail address: agol@math.uic.edu

Current address: Department of Mathematics, University of California at Berkeley, 970 Evans

Hall \#3840, Berkeley, California 94720-3840

E-mail address: ianagol@gmail.com

Department of Mathematics, Stanford University, Bullding 380, 450 Serra Mall, Stanford, California 94305-2125

E-mail address: storm@math.stanford.edu

Department of Mathematics, Cornell University, 310 Malott Hall, Ithaca, New YORK 14853-4201

E-mail address: wpt@math.cornell.edu 
Department of Mathematics, 253-37, Caltech, Pasadena, California 91125

E-mail address: dunfield@caltech.edu

Current address: (August 1, 2007) Department of Mathematics, University of Illinois at Urbana-Champaign, 1409 W. Green Street, Urbana, Illinois 61801

E-mail address: nathan@dunfield.info 